\title{
High-Accuracy Simulations to Model Pyrometallurgical Processes in a Secondary Lead Reverberatory Furnace
}

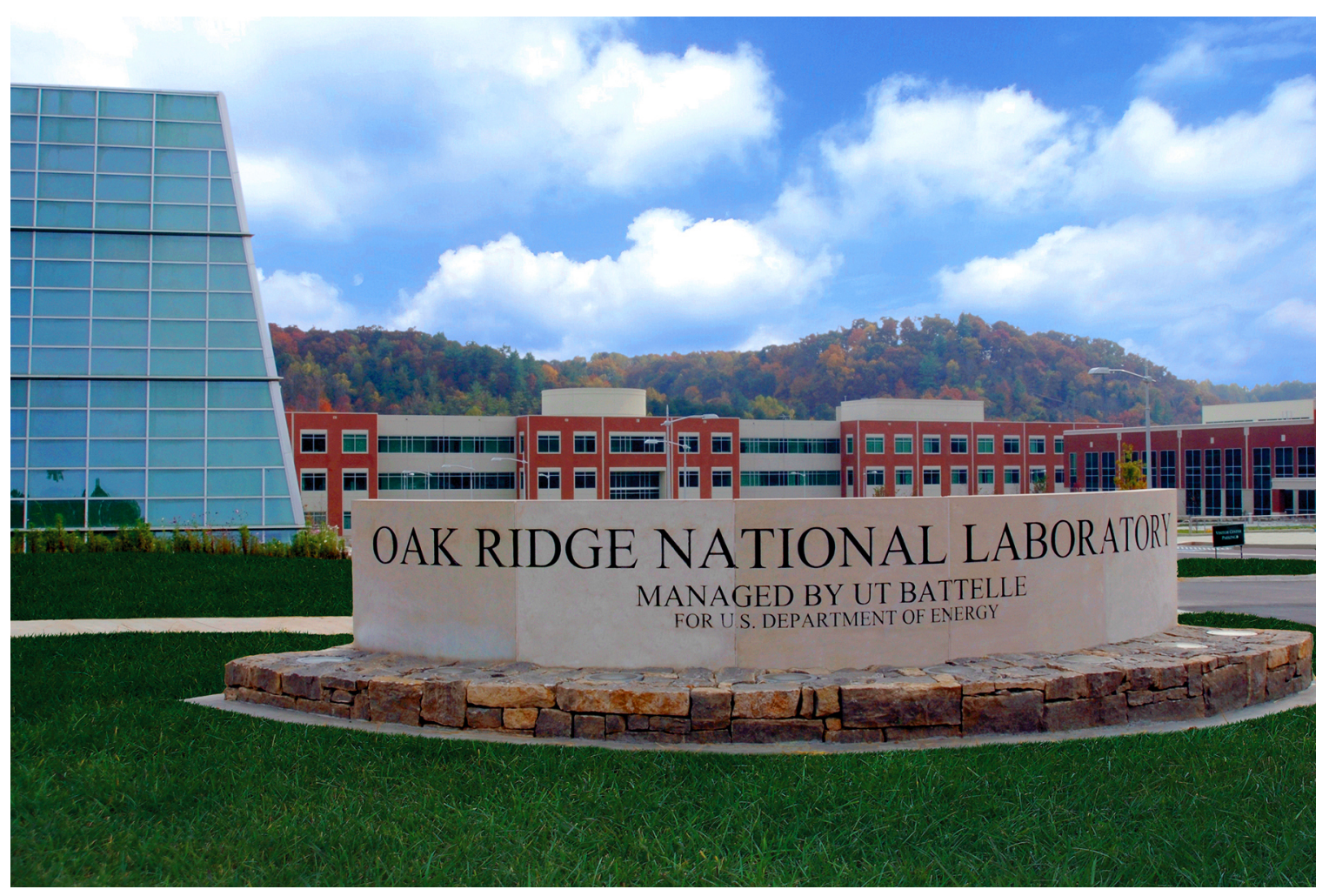

Vivek M. Rao

Vineet Kumar

Alexandra Anderson

Prashant K. Jain

August 31, 2021

Approved for public release. Distribution is unlimited. 


\section{DOCUMENT AVAILABILITY}

Reports produced after January 1, 1996, are generally available free via US Department of Energy (DOE) SciTech Connect.

Website: www.osti.gov/

Reports produced before January 1, 1996, may be purchased by members of the public from the following source:

National Technical Information Service

5285 Port Royal Road

Springfield, VA 22161

Telephone: 703-605-6000 (1-800-553-6847)

TDD: $703-487-4639$

Fax: 703-605-6900

E-mail: info@ntis.gov

Website: http://classic.ntis.gov/

Reports are available to DOE employees, DOE contractors, Energy Technology Data Exchange representatives, and International Nuclear Information System representatives from the following source:

Office of Scientific and Technical Information

PO Box 62

Oak Ridge, TN 37831

Telephone: 865-576-8401

Fax: 865-576-5728

E-mail: report@osti.gov

Website: http://www.osti.gov/contact.html

This report was prepared as an account of work sponsored by an agency of the United States Government. Neither the United States Government nor any agency thereof, nor any of their employees, makes any warranty, express or implied, or assumes any legal liability or responsibility for the accuracy, completeness, or usefulness of any information, apparatus, product, or process disclosed, or represents that its use would not infringe privately owned rights. Reference herein to any specific commercial product, process, or service by trade name, trademark, manufacturer, or otherwise, does not necessarily constitute or imply its endorsement, recommendation, or favoring by the United States Government or any agency thereof. The views and opinions of authors expressed herein do not necessarily state or reflect those of the United States Government or any agency thereof. 
ORNL/TM-2021/2129

CRADA/NFE-19-07865

Nuclear Energy and Fuel Cycle Division

\title{
High-Accuracy Simulations to Model Pyrometallurgical Processes in a Secondary Lead Reverberatory Furnace
}

\author{
Authors \\ Vivek M. Rao \\ Vineet Kumar \\ Alexandra Anderson \\ Prashant K. Jain
}

Date Published: August 31, 2021

\author{
Prepared by \\ OAK RIDGE NATIONAL LABORATORY \\ Oak Ridge, TN 37831-6283 \\ managed by \\ UT-Battelle, LLC \\ for the \\ US DEPARTMENT OF ENERGY \\ under contract DE-AC05-00OR22725
}





\section{CONTENTS}

LIST OF FIGURES $\ldots \ldots \ldots \ldots \ldots \ldots \ldots \ldots \ldots \ldots \ldots$

LIST OF TABLES $\ldots \ldots \ldots \ldots \ldots \ldots \ldots \ldots \ldots \ldots$ vii

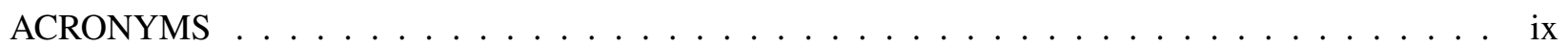

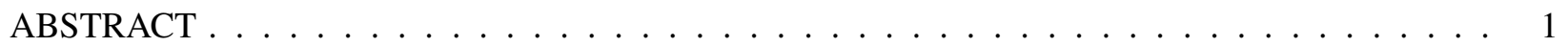

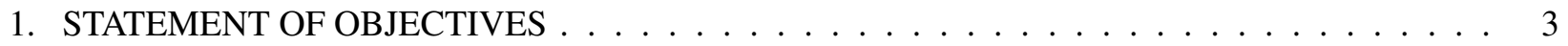

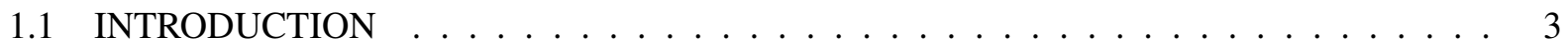

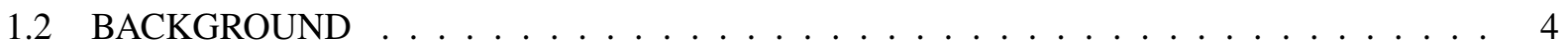

1.3 CAPABILITIES AND LIMITATIONS OF THE CURRENT STATE OF THE ART $\ldots \ldots$

2. BENEFITS TO THE FUNDING DOE OFFICE'S MISSION . . . . . . . . . . . . . . . . . . 10

3. TECHNICAL DISCUSSION OF WORK PERFORMED BY ALL PARTIES $\ldots \ldots \ldots$

3.1 BURNER AND GAS FLOW FIELD MODELING AND VALIDATION $\ldots \ldots \ldots$

3.1 .1 Eddy Break-Up Model Description in STAR-CCM+ . . . . . . . . . . . . . . 11

3.1.2 Chemical Kinetics Scheme for Oxy-Fuel Combustion of Natural Gas . . . . . . . . 14

3.1 .3 Other relevant models used in STAR-CCM $+\ldots \ldots \ldots \ldots \ldots$

3.1.4 Model Validation of Gopher Resource Furnace Measurements . . . . . . . . . . . . 17

3.2 THERMAL AND HYDRODYNAMICS INVESTIGATION OF LAGRANGIAN

PARTICLE LOADING . . . . . . . . . . . . . . . . . . . . . 25

3.2.1 Lagrangian/DEM Model Description in STAR-CCM+ . . . . . . . . . . . . . 25

3.2.2 Closure Models and other Relevant Models Used in LMP . . . . . . . . . . . . . . . 27

3.2.3 Lagrangian and DEM Modeling Runs . . . . . . . . . . . . . . . . . . . . . . . . . . . .

3.3 MULTI-PHYSICS SIMULATION OF THE WHOLE FURNACE . . . . . . . . . . . . . 34

3.3.1 Relevant Mathematical Models for Full-Furnace Simulation in STAR-CCM+ . . . . 34

3.3.2 Other Closure Models for the Full-Furnace Simulation in STAR-CCM+ . . . . . . . 36

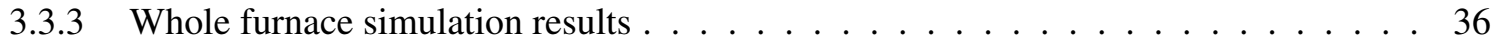

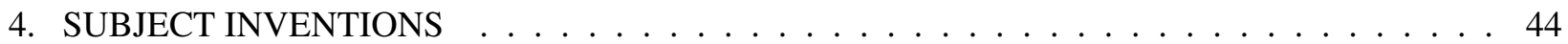

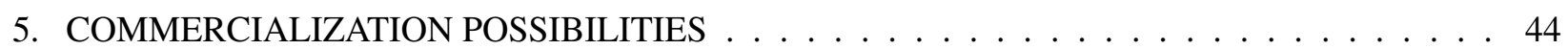

6. PLANS FOR FUTURE COLLABORATION . . . . . . . . . . . . . . . . 44

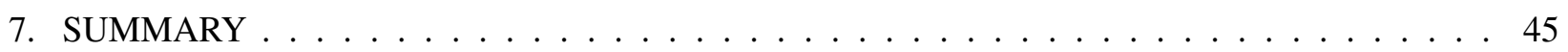

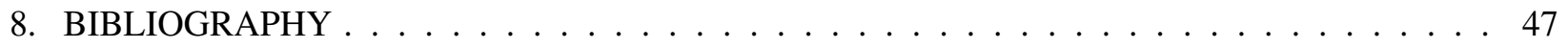





\section{LIST OF FIGURES}

1 A typical direct style reverberatory furnace for the secondary lead processing (from osha.gov) 4

2 Phase(s) distribution in a secondary $\mathrm{Pb}$ furnace. . . . . . . . . . . . . . . 5

3 A detailed snapshot of the complex multiphysics interactions in a secondary $\mathrm{Pb}$ furnace. $\quad . \quad 5$

4 Image of the inside of the test furnace showing views of the cooling tubes, floor plates, and back wall panels. . . . . . . . . . . . . . . . . . . . 17

5 Quality assurance process to optimize the mesh of a CFD model. . . . . . . . . . . . 18

6 Mesh snapshot of the test furnace showing the well-resolved burner region. . . . . . . . . . 19

7 Furnace geometry showing the spherical igniter colored purple. . . . . . . . . . . . . . . 19

8 Furnace measurement validation and combustion flow field simulation. . . . . . . . . . . 23

9 Validation of experimental data for a chosen condition. . . . . . . . . . . . . . 24

10 Cold-flow simulation: (top view) effect of plume momentum on an initially static burden of true composition. . . . . . . . . . . . . . . . . . . . . 33

11 Normalized combustion model results for the whole furnace steady state operation. . . . . . 38

12 Distribution of parcels in the reverberatory furnace . . . . . . . . . . . . . . . . 39

13 Thermal response of feed to heat from combustion. . . . . . . . . . . . . . . . 40

14 Variation of feed density caused by thermal effects. . . . . . . . . . . . . . . 40

15 Slip velocity of feed parcels in the flue flow field . . . . . . . . . . . . . . . 41

16 Variation of species within parcels due to thermal effects. . . . . . . . . . . 43 



\section{LIST OF TABLES}

1 A summary of capabilities when model sets are simultaneously activated in STAR-CCM+ . 6

2 Jones-Lindstedt (JL) four-step reaction mechanisms for methane with the kinetic rate data . 14

3 JL four-step reaction mechanisms for ethane with the kinetic rate data . . . . . . . . . . 15

4 JL four-step reaction mechanisms for propane with the kinetic rate data $\ldots \ldots \ldots$

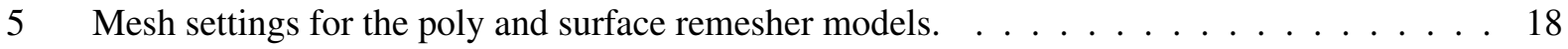

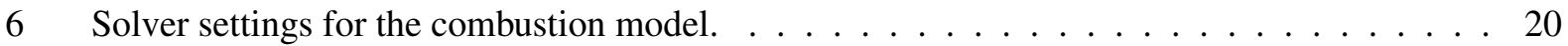

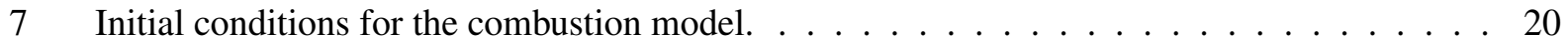

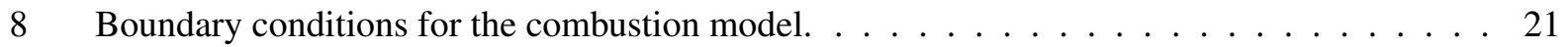

9 Normalized burner set point for the validated combustion model. . . . . . . . . . . . . . 21

10 Parallel processing settings for the lagrangian simulation runs . . . . . . . . . . . . . 22

11 Solver settings for the Lagrangian multiphase flow (LMP) model . . . . . . . . . . . . 31

12 Initial conditions for the LMP model . . . . . . . . . . . . . . . . . . . 32

13 Parallel processing settings for the lagrangian simulation runs . . . . . . . . . . . . . 32

14 Solver settings for the whole furnace model . . . . . . . . . . . . . . . . . . 37

15 Initial conditions for the combustion model . . . . . . . . . . . . . . 37

16 Parallel processing settings for the final whole furnace simulation . . . . . . . . . . . . 37 



\section{ABBREVIATIONS}

CFD computational fluid dynamics

CO carbon monoxide

$\mathrm{CO}_{2}$ carbon dioxide

CPU central processing unit

DEM discrete element method

DOE Department of Energy

EBU eddy break-up

EMP Eulerian multiphase

GTI Gas Turbine Institute

HPC high-performance computing

HPC4Mfg High-Performance Computing for Manufacturing Program

JL Jones-Lindstedt

LMP Lagrangian multiphase flow

MSF multiphase segregated flow

OFC oxy-fuel combustion

ORNL Oak Ridge National Laboratory

$\mathbf{P b}(\mathbf{O H})_{2}$ lead hydroxide

$\mathrm{PbCO}_{3}$ lead carbonate

PbO lead oxide

PbS lead sulfide

$\mathbf{P b S O}_{4}$ lead sulfate

$\mathbf{S O}_{2}$ sulfur dioxide

VOF volume of fluid 



\begin{abstract}
The US manufacturing industry produces about 1.3 million tons of refined lead each year using secondary sources consisting mainly of lead batteries. Oak Ridge National Laboratory is partnering with Gopher Resource - the second largest lead recycling company in the United States - and the Gas Turbine Institute to develop a high-fidelity computational fluid dynamics (CFD) model of a directly fired, reverberatory-style secondary lead furnace. These high-performance computing (HPC) simulations aim to use first-principles modeling for combustion and melting processes of the secondary lead feed while accounting for complex interphase interactions between the gas, solid charge material (lead), slag, and metal phases. By performing validation against operating plant data, this effort will enable significant improvements in design, operational parameters, and energy efficiency, thus improving the productivity and refractory lifetime of secondary lead melting furnaces. The US lead industry can expect an estimated savings/reduction of at least 1 trillion Btu per year, 1 million tons of greenhouse gas emissions per year, and $\$ 50$ million per year. ORNL resources and expertise in HPC and multicomponent, multiphase flows were utilized to realize this modeling goal while advancing the understanding of the smelting and melting processes occurring within the furnace.

During the first stage, a CFD model of an experimental furnace was constructed to validate tests conducted by Gopher Resource. The tests were conducted using Gopher's proprietary burners to comprehensively validate the CFD combustion model. These tests included thermocouple measurements at various locations in the furnace, in-flame carbon monoxide (CO) measurements, and flue composition, which were conducted for different flow boundary conditions. During the second stage of the project, the hydrodynamics of the solid feed particles were investigated with the validated combustion model for various inlet velocities and various uniform particle sizes. Sensitivity studies were conducted using two different Lagrangian modeling approaches. The discrete element method (DEM) was shown to capture the particle dynamics reasonably well in terms of its comparison with images of the actual furnace operation. The third and final stage of the modeling effort focused on simulating the whole furnace operation, which included the combustion flow field, solid particle loading, smelting and melting processes, and formation of slag and lead. Some of the difficulties encountered at this stage were the lack of smelting kinetics data - which had to be obtained/inferred from various sources, as well as workarounds for simulating melting physics of solid Lagrangian/DEM particles_-and the transfer of the solid feed particles to a continuum fluid model at their melting points. The final stage was accompanied by a verification and validation effort that included the comparison of the simulation predictions with plant data for steady-state operations for the flue gas emissions and amount of molten lead flowing out of the lead tap.
\end{abstract}





\section{STATEMENT OF OBJECTIVES}

\subsection{INTRODUCTION}

Advances in the extraction and production of metals are critical because they are key drivers of global industrial growth, and advancement of these processes is also crucial for mitigating their significant annual carbon footprint. The world's major metals- $\mathrm{Fe}, \mathrm{Al}, \mathrm{Mn}, \mathrm{Cu}, \mathrm{Zn}, \mathrm{Pb}$, and $\mathrm{Ni}-$ constitute $98 \%$ by mass of the annual industrial production globally $[1,2]$. In terms of future global growth/demand forecasts up to the end of this century, relative to 2010, the largest growth rates are predicted to be for $\mathrm{Al}(470 \%)$ followed by $\mathrm{Cu}(330 \%), \mathrm{Zn}(130 \%)$, and $\mathrm{Fe}(110 \%)$ [1]. Lead production is expected to grow until 2050, and then there is a predicted decrease of global $\mathrm{Pb}$ demand beyond $2050[3,1]$. According to the International Lead and Zinc Study Group [4], global refined lead production reached $\sim 11.7$ million tonnes (mt) ( $\sim 62 \%$ secondary lead production) in 2018 , and the majority of the world's production $(\sim 42 \%)$ took place in China [4]. Of the nonferrous metals, the five that produce the highest annual carbon dioxide $\left(\mathrm{CO}_{2}\right)$ emissions from production in China are $\mathrm{Al}, \mathrm{Cu}, \mathrm{Pb}, \mathrm{Zn}$, and $\mathrm{Mg}$, which together constitute $94 \%$ of the total $\mathrm{CO}_{2}$ emissions generated by the nonferrous metals industry between 2010 and 2015 [5]. Given the projections for $\mathrm{CO}_{2}$ emissions, there is a greater need to improve the production of $\mathrm{Pb}$, primarily secondary $\mathrm{Pb}$, which accounts for the bulk of the global production and an even greater share in the United States.

Pyrometallurgical furnaces remain an integral part of the processing route for many nonferrous metals, including $\mathrm{Pb}$. The inner workings of the furnaces are very complex. Direct observation and process measurements can provide some degree of insight but are often very challenging to carry out in the high-temperature, corrosive environments inherent to these types of operations. Even with advancements in direct measurement techniques, the multiphase heat and mass transport phenomena and complex chemical reactions involved make these furnaces difficult to deterministically control. These processes often involve media that undergo various phase changes and several types of reactions-such as decomposition, reduction, oxidation, and slagging - that are influenced not only by the material itself but also by other furnace conditions. Furthermore, pyrometallurgical furnaces suffer from low efficiencies if they are not designed or operated optimally, and they generate toxic emissions such as sulfur dioxide $\left(\mathrm{SO}_{2}\right)$, nitrous oxide (NOx), carbon monoxide (CO), and arsenide. Current energy efficiencies in these furnaces can still be as low as $30 \%$, and over $70 \%$ of the energy can be lost during the material processing. Over the past several decades, tremendous effort has gone into improving the energy efficiency of nonferrous metal production, particularly in the $\mathrm{Al}$ industry. Previous studies of this industry have shown that optimally designed reverberatory-style furnaces can operate at improved efficiencies of $40 \%$ or greater [6], which is a promising result to work toward for the nonferrous metals industry as whole.

Some standard methodologies for designing and investigating nonferrous pyrometallurgical furnaces are empirical analysis, simulation of experiments based on similarity theory, thermodynamic and kinetic modeling, and numerical analysis [7, 8]. Often, a combination of these techniques is used. Numerical modeling has proven to be an important tool, helping achieve improvements in furnace design and operation alongside the advances in experimental data acquisition [9]. However, the challenging environments in pyrometallurgical furnaces limit the scope of further furnace instrumentation. Advances in high-resolution computational fluid dynamics (CFD) codes can help fill the gaps in our understating of the complex thermophysical process in these furnaces toward realizing the goals of improved energy efficiency, and consequently, reduction in the carbon footprint. The direct-style reverberatory furnace is typically used in modern secondary $\mathrm{Pb}$ processing plants (shown in Fig. 1). A combination of a turbulent flame from 


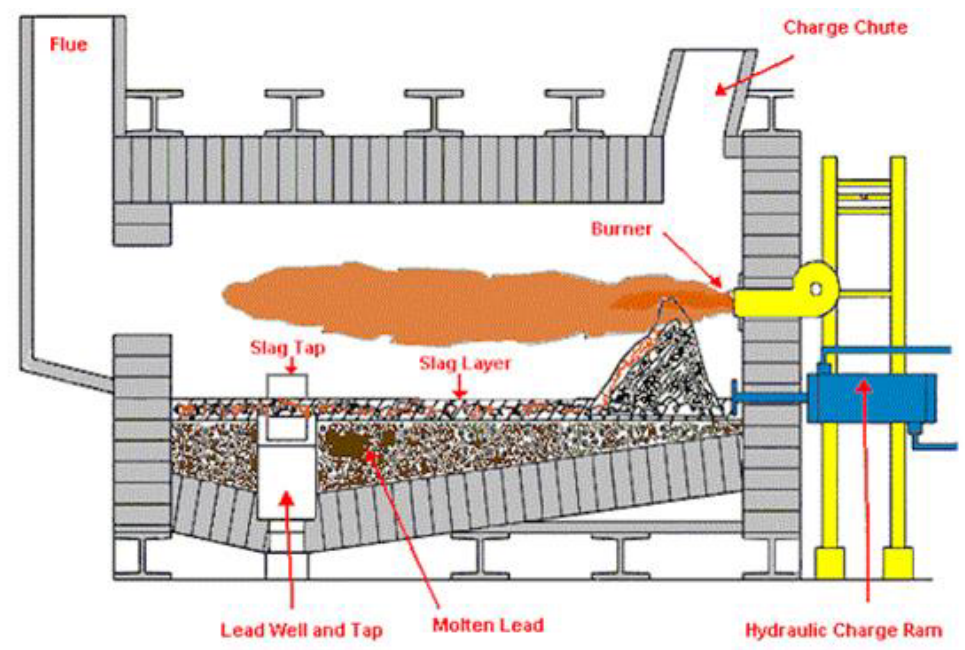

Figure 1. A typical direct style reverberatory furnace for the secondary lead processing (from osha.gov)

accelerating combustion gases and radiant heating, generate high amounts of heat which are directed to the burden. However, the high-velocity, high-temperature gas flow can produce high wall shear stress and hot spots on the refractory lining. These phenomena can lead to excessive erosion and thermal stress, thereby prematurely shortening the lifetime of the refractory. These types of furnaces can also suffer from low efficiencies if they are not designed or operated optimally. As mentioned previously, current energy efficiencies are still less than $30 \%$ as an industry standard, with over $70 \%$ of the energy lost during the melting processes. By using the learnings from the aluminum industry, there is significant potential to improve the energy efficiencies of reverberatory lead furnaces, particularly using high-resolution CFD modeling run on high-performance computing (HPC) clusters. It is projected that the resulting improvements can provide energy savings of at least 1 trillion Btu per year, reductions in greenhouse gas emissions of at least 1 million tons per year, and a total cost savings of more than $\$ 50$ million per year to the US lead industry.

\subsection{BACKGROUND}

Secondary $\mathrm{Pb}$ production typically involves the recycling of spent lead-acid batteries. Although the details of the lead-acid battery recycling process can vary from one processing plant to the next, the general sequence of unit processes remains fairly consistent. The batteries are first drained of acid and the outer casing is removed manually or by crushing. A series of physical and chemical separation techniques are then performed to produce a $\mathrm{Pb}$ concentrate [10]. The $\mathrm{Pb}$ bearing material is then smelted with coke and other fluxes in single furnaces or a combination of rotary, reverberatory, or blast furnaces. The smelting process is followed by a refining process by which unwanted impurities are removed from the $\mathrm{Pb}$ through preferential oxidation. The final $\mathrm{Pb}$ product is then sent to casting operations [11, 12].

One of the major challenges when modeling secondary $\mathrm{Pb}$ reverberatory furnaces is the treatment of the interface between the combustion gases and the furnace burden, which consists of a solid heterogeneous mix of $\mathrm{Pb}$-bearing compounds and fluxes, a molten bath, a slag phase, and a $\mathrm{Pb}$ metal phase [13]. Fig. 2 
depicts a schematic of the approximate distribution of these phases in a typical $\mathrm{Pb}$ reverberatory furnace. Solid charge material is fed into the end wall on the burner side of the furnace. Most of the melting and smelting reactions occur in the front half of the furnace, which produces a molten bath below the solid charge. Separation of the resulting metal and slag layers occurs in the back half of the furnace. The combustion gases reside above the solid feed phase and slag layer, and they exit through the flue.

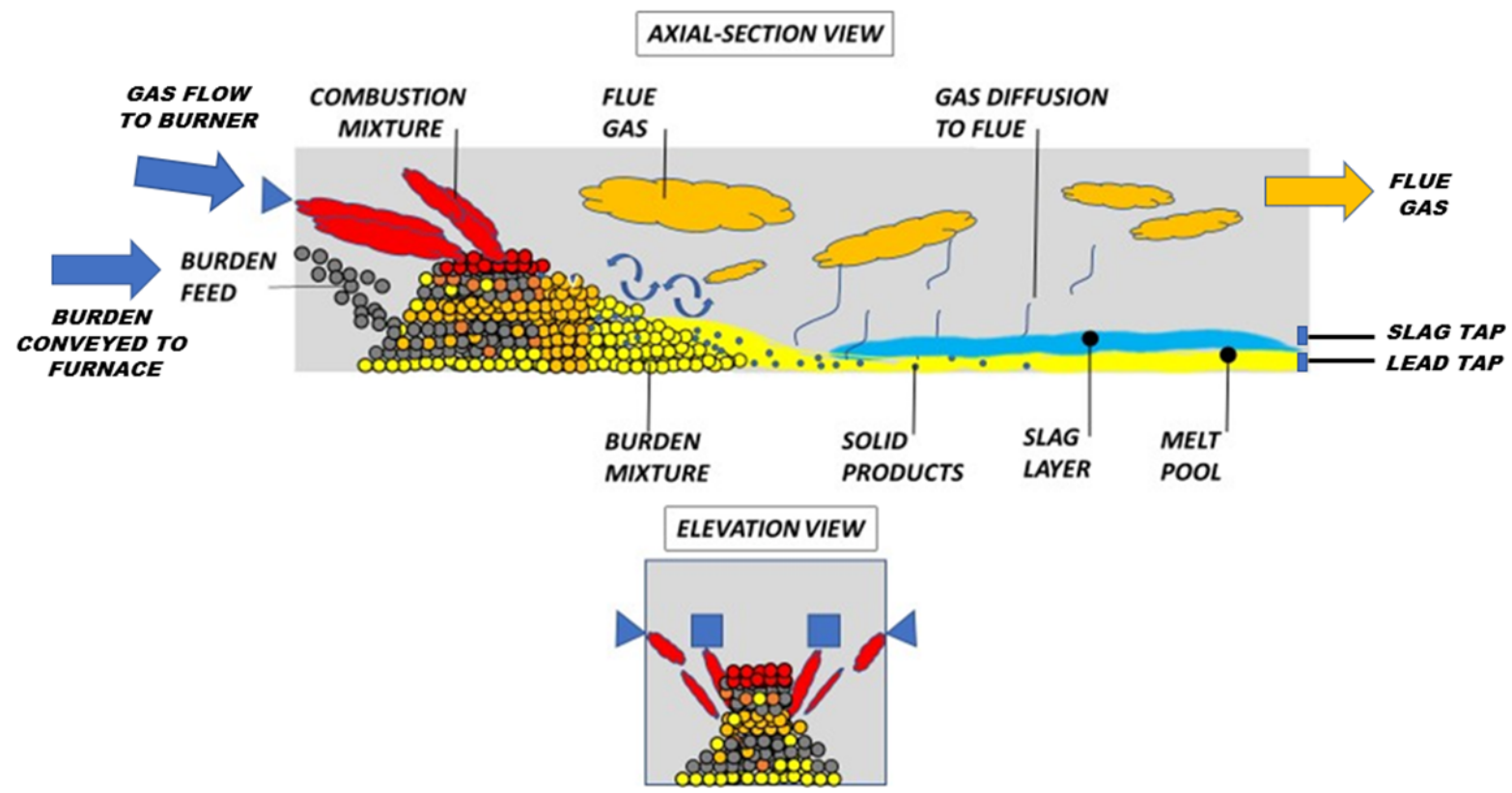

Figure 2. Phase(s) distribution in a secondary Pb furnace.

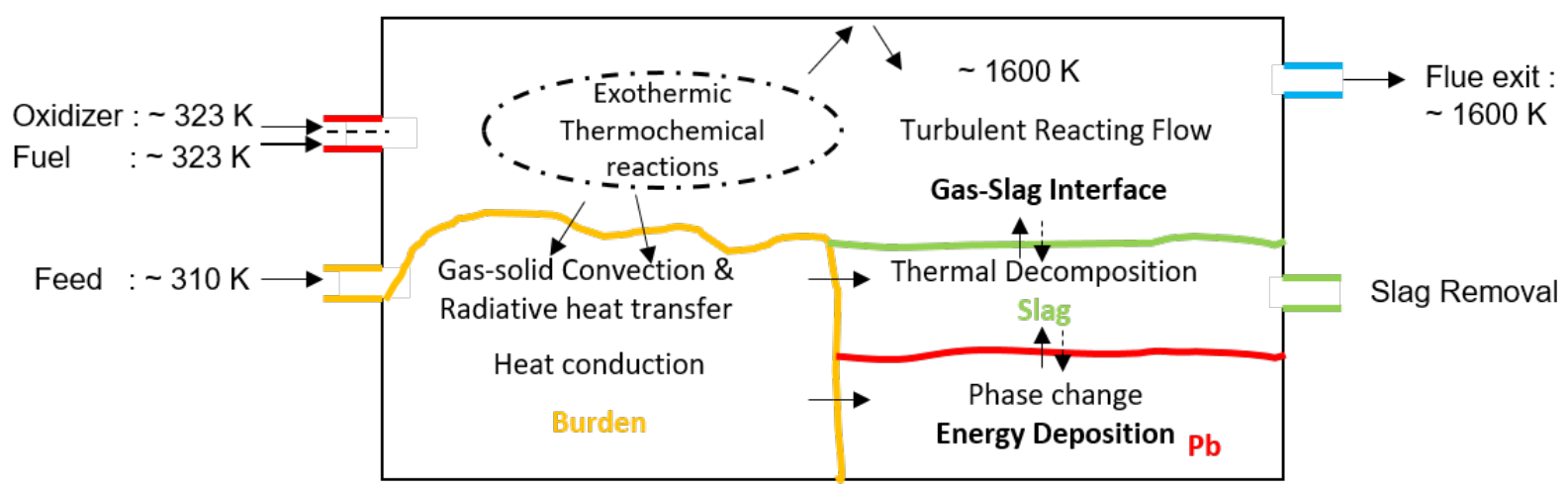

Figure 3. A detailed snapshot of the complex multiphysics interactions in a secondary Pb furnace.

Only a handful of scientific publications discuss the mechanism of the gas-burden interface and metal phase transformation for applied CFD in the pyrometallurgical industry as a whole. Within the pyrometallurgical space, the modeling community has directed most of its effort toward $\mathrm{Al}$ and $\mathrm{Cu}$, and there is a paucity of literature on $\mathrm{Pb}$. Current modeling efforts of secondary $\mathrm{Pb}$ furnaces have accounted for the consumption of heat from the smelting and melting reactions by defining a constant heat sink with steady-state simulations above the gas-burden interface $[14,13]$. Although this approach allows the CFD model to inform the design or operational changes based on the distributions within the gas region, it limits 
the model's ability to predict the impact that these changes might have on furnace efficiency or productivity. This is because the simulation does not provide any resolution regarding the consumption of the heat by the solid burden and therefore, cannot predict the actual production of secondary aluminum or lead without using additional empirical models/correlations. Furthermore, this treatment of the gas-burden interface could lead to inaccuracies caused by not fully accounting for melting, heat loss, phase change of the charge material, and so on, as detailed in Fig. 3. Development of a robust, high-fidelity method for resolving the interaction between the gas space and furnace burden regions will allow CFD modeling efforts to produce information on furnace efficiency and productivity as a function of design changes.

\subsection{CAPABILITIES AND LIMITATIONS OF THE CURRENT STATE OF THE ART}

STAR-CCM+ (Version 2021.1 Build 16.02.008) from Siemens [15] is an American Society of Mechanical Engineers (ASME) Nuclear Quality Assurance (NQA-1)-compliant finite-volume CFD solver, and it has been extensively benchmarked and applied to various areas such as in multiphase flows, external aerodynamics, reacting flow networks, and treatment of complex geometric shapes [16].

The recovery of secondary $\mathrm{Pb}$ poses unique challenges in an added layer of physicochemical complexity - heterogeneous chemical reactions (solid to solid and gas), melting, and diffusion of gases through the free surface flow of molten $\mathrm{Pb}$, as shown in Fig. 2. The furnace components consist of a Gas phase that refers to the mixture of gaseous reactants and products, a solid Burden phase which refers to a mixture of secondary $\mathrm{Pb}$ feed that contains both desired and undesired products, and a Melt pool which refers to the volume of molten $\mathrm{Pb}$, which can be treated as a non-Newtonian, radiating fluid. While the Gas phase can be treated a reacting Eulerian multi-component mixture, the solid Burden would have to be treated as a particulate Lagrangian multi-component mixture to resolve particle-particle, and particle-gas interfaces as well as account for thermal decomposition. The Melt pool can be treated as a continuous fluid phase with relevant properties or a Lagrangian liquid. In STAR-CCM+, many models are developed with a specific range of applications in mind, which allows for simulation of simultaneous and competing effects. The following discussion explains the capabilities and limitations of the different approaches listed in Table 1 using STAR-CCM+ to evaluate permutations of models that might best describe thermophysical and chemical phenomena that occur in the recovery process of $\mathrm{Pb}$. It must be noted that in Table 1, the treatment of materials is a combination of mixtures and singular phases.

Table 1. A summary of capabilities when model sets are simultaneously activated in STAR-CCM+

\begin{tabular}{|c|c|c|c|c|c|c|}
\hline \multirow{2}{*}{ Model category } & \multicolumn{3}{|c|}{ Phases } & \multicolumn{3}{|c|}{ Thermophysical phenomena } \\
\hline & Gas phase & Burden & Melt pool & OFC & Thermal decomp. & Melt pool formation \\
\hline 1 & MSF & MSF & MSF & Yes & Yes & No \\
\hline 2 & VOF & VOF & VOF & Yes & Yes & No \\
\hline 3 & VOF & VOF M-S & VOF M-S & Yes & No & No \\
\hline 4 & VOF & LMP & VOF & Yes & No & No \\
\hline 5 & VOF & LMP-DEM & VOF & Yes & No & Yes \\
\hline 6 & VOF & Fluid film & VOF & Yes & Yes & No \\
\hline 7 & Mixture & LMP & LMP & Yes & Yes & No \\
\hline 8 & Mixture & LMP-DEM & LMP & Yes & Yes & Yes \\
\hline
\end{tabular}


1. All phases here may be treated with an Eulerian multiphase (EMP) formulation using the multiphase segregated flow (MSF) model, in which transport equations are solved for each phase. The gas phase contains all gaseous species. The burden contains all solid species. The melt pool is a single-liquid phase. The isolated models per phase allow for greater accuracy in the simulation of phase-specific effects at the cost of higher computational expense, which is caused by the greater number of solved equations. Capabilities: This setup allows for the simulation of gas combustion models based on eddy dissipation and turbulent flow interactions. The burden and gas participate in an interphase interaction set of models to decompose the solid components in the burden into simpler solids and gases that unite with the gas mixture. Limitations: There is no established interphase interaction to simulate the melting of simpler solids in the burden to components in the melt pool.

2. In the volume of fluid (VOF) approach, the mixture of gas species is assigned as a single phase, and the burden and melt pool are assigned to a single phase. This is done because a model for melting and solidification was developed for fluids within the VOF framework. Each VOF phase is treated as a singular phase, and the volume fractions of the phases are conserved to unity. Within each phase, the components can be single materials or mixtures. If the components are a mixture, the mass fraction is intrinsically conserved to unity within the VOF phase. Capabilities: The burden and liquid exist within a single VOF phase using the melting point (or solidus and liquidus) as a criterion to determine the formation and existence of solid and liquid states of the material. This approach allows for simple predictions when simulating the melt pool formation from single metallic solids. As with case (1), this setup allows for the simulation of oxy-fuel combustion (OFC) of the gas mixture within the VOF phase, and the heat from OFC is used to sustain the heating of the solid component in the burden to the melting point, absorption of the latent heat (of fusion), and further heating of the liquid component formed in the melt pool. The prevailing local temperature dictates which state of the material exists in the solid-liquid VOF phase, allowing interphase interactions between this phase and the gaseous VOF phase. This capability is ideal for simulating the use of combustion to melt simple metals into their molten phases. Limitations: The melting-solidification capability was developed only for single components. In the recovery of secondary $\mathrm{Pb}$, the solids that melt must first be formed by the thermal decomposition of larger solids, using heat from OFC. This process requires the assignment of source solids to a burden mixture, which renders them incompatible for use within the same phase as the melt pool.

3. This combination is a variant of case (2) that is applicable to simulation of the melting and solidification of multiple single metallic components in the burden. Thus, the heat from OFC could be used by multiple metallic components to increase their temperatures to the melting point and transition to the molten phase-provided that there is sufficient heat available for fusion. This approach would be suitable for materials the solid and liquid states of which could be assigned to a single VOF phase, but not for solids that must decompose before the melting of their products.

4. In the Lagrangian multiphase flow (LMP) approach, the burden is modeled as cumulative masses of discrete particle aggregates that can be injected through physical or artificial boundaries assigned to the physical domain. Artificial boundaries such as trap walls are often used to increase computational speed or develop a stable initial solution before solving for phenomena with high-resolution effects. An advantage of the LMP formulation over the EMP framework is the inherent difference in the reference frame: a phase assigned to the LMP framework can be tracked in its trajectory and time throughout the physical domain. This approach offers significant insight into the behavior of a particular aggregate of particles, or even a single particle, over specific domains of 
interest, such as the thermal response of particles in the burden to impinging flames from OFC and the distribution of high-temperature zones in the burden along the length of the reverberatory furnace. Capabilities: When the LMP approach is combined with Eulerian VOF phases, the gas phase combustion can be modeled via eddy dissipation and turbulence interactions within a VOF phase. Moreover, the response of the LMP particles to prevailing Eulerian effects in high spatial resolution can be studied in terms of momentum and energy via two-way coupling. A VOF phase can be assigned to a material the solid-liquid pair of which needs no chemical transformation before or after absorbing/releasing (respectively) the latent heat of fusion. Limitations: The LMP formulation is inherently incompatible with an EMP formulation when mass transfer is considered. There is no conservation available between the phases through chemical reactions. Therefore, the burden cannot decompose into gases because the gases are assigned to an EMP phase via VOF. Additionally, in the absence of thermal decomposition, the resulting product cannot interact with its molten state in the other VOF phase.

5. This case is an extension of case (4), and in this case, the discrete element method (DEM) model is used to capture high-resolution effects in space and time for particle-laden flows. Historically, the DEM model has been used in the simulation of molecular-scale dynamics and can provide otherwise impossible-to-measure detail on particle-wise values of temperature, velocity, and concentration, among other parameters. The DEM model is extensively used in the pharmaceutical industry, in drying applications [17], [18], and in seeded particulate flows, in which the volume fraction of particles is very low and yet has significant effects on the local flow field. With the DEM model, additional models for rolling resistance, artificial viscosity, particle-particle collisions, and particle-wall collisions provide valuable detail to pyrometallurgical applications for discerning injection effects on the burden in reverberatory furnaces. However, as in case (4), the incompatibility of the LMP framework with VOF phases in mass-transfer applications prevents this combination of models from simulating all effects of interest.

6. In this combination, the burden is assigned to a "fluid film" model. The fluid film can host a liquid, a gas, a solid-liquid-gas mixture, or a multicomponent liquid mixture as a single phase. This model is suitable for film-dominant applications. A film forms as a result of surface tension and is driven by laminar flow or a film breaks into discrete droplets because of shear effects from ambient turbulence. Capabilities: The fluid film model is fully compatible with VOF phases. It can decompose a solid-liquid-gas mixture using the heat of combustion from the ambient gaseous VOF phase via interphase interactions. The gaseous products from thermal decomposition of the burden can be transferred to the gaseous VOF phase using interphase interactions. Limitations: The solids formed by thermal decomposition within the fluid film phase belong to a mixture and can interact only with liquid mixtures in the VOF phase. Furthermore, such an interphase transfer of mass would nonphysically transfer all solid components from the fluid film to the mixture of liquids within the VOF phase. This transfer is not appropriate for recovery processes because materials melt at temperatures that are hundreds of degrees apart and form free surfaces at different locations. Therefore, this combination is not suitable and is incapable of capturing the desired effects.

7. In this combination, the gas phase is assigned to an Eulerian mixture model, and the burden and the melt pool use the LMP approach. This combination of models allows for thermal decomposition of the burden phase and smelting reactions (with some caveats), as well as transfer of the emitted gaseous products to the gas phase. The drawback for the Eulerian mixture model is that only one momentum equation - and thus only one mixture velocity—is solved for all the gaseous 
components. Furthermore, parcel transfer injectors can be used to transfer between Lagrangian phases (i.e., from the solid burden to the melt pool at the melting temperature). This combination cannot simulate detailed melting physics. Capabilities: The LMP model can simulate the thermal decomposition of the burden and the transfer of products to the gaseous Eulerian mixture phase, including multicomponent melting via parcel transfer injectors. Interphasic interactions between the LMP particles and the gas phase can be accounted for, such as drag, interphasic heat transfer, and turbulent dispersion. Additionally, the melt pool includes models such as impingement heat transfer and droplet-to-film transition. Limitations: Flocculation of the Lagrangian particles by the gas phase makes this combination of models unsuitable. Therefore, rigid DEM particles are required to avoid fluidization of the Lagrangian particles into the gas stream.

8. This case is a mixture of cases (5) and (7). Here, the burden phase is a combination of LMP and DEM so that fluidization into the gas stream can be avoided, and the gas phase is the Eulerian multicomponent mixture model. As in case (7), thermal decomposition of the burden phase can be simulated, including transfer of gases to the Eulerian multicomponent mixture phase. Capabilities: The LMP-DEM models can simulate the thermal decomposition of the burden and transfer of products to the gaseous Eulerian mixture phase, including multicomponent melting via parcel transfer injectors. Additionally, DEM includes a catalog of differently shaped particles beyond spherical particles, as previously mentioned. The DEM model can also simulate interparticle and particle-wall interactions using models such as conduction heat transfer and Hertz-Mindlin contact models. Limitations: Multireactant smelting reactions allow only for user reaction rates instead of kinetic data, which is a significant limitation of this model. The other major limitation is that reactions are allowed only for multicomponent particles and not between two different particles. Finally, transfer of the solid burden to the VOF phase would be ideal to simulate stratification effects between the slag and melt pool, as well as to simulate slag-flue gas interactions. This limitation is partially alleviated by the fact that the LMP melt pool allows interphasic interactions such as drag and turbulent dispersion.

Although case (8) appears to be the most promising approach, the actual code performance would have to be tested for this set of models to ensure that it is able to simulate the relevant physics. Furthermore, as development of multiphase CFD solvers increases, some of the models that are currently incompatible could be used in conjunction with each other, such as the transfer of solid LMP or DEM particles to VOF. In this study, a variation of case (8) is used to overcome some of its limitations, such as particle flocculation on transfer of solid feed to liquid melt. More investigation is required to study how a continuous (Lagrangian) liquid melt pool could be simulated with this approach. 


\section{BENEFITS TO THE FUNDING DOE OFFICE'S MISSION}

The High-Performance Computing for Manufacturing Program (HPC4Mfg) unites the world-class computing resources and expertise of US Department of Energy (DOE) national laboratories with US manufacturers to deliver solutions that could revolutionize manufacturing. Led by Lawrence Livermore National Laboratory and joined by principal national laboratories, HPC4Mfg offers a low-risk path for manufacturing companies interested in adopting HPC to advance clean energy technologies and increase energy efficiency.

The DOE Advanced Manufacturing Office (AMO) within the Energy Efficiency and Renewable Energy Office sponsors this program. Through HPC4Mfg, industrial partners collaborate with national laboratory experts in advanced modeling, simulation, and data analysis on project teams. These teams address process optimization and design, quality improvement, performance and failure prediction, accelerating or eliminating testing, shortening time to adoption, and decision-making.

This project with Gopher Resource and Gas Turbine Institute (GTI) helped to realize the following benefits in support of the DOE mission:

- Infuse advanced CFD computing expertise and technology into the secondary lead industry

- Develop advanced numerical CFD capabilities for highly resolved multiphase simulations

- Develop new modeling pathways for simulating all the relevant physics in secondary $\mathrm{Pb}$ furnaces

- Strengthen industrial competitiveness through advanced research and development 


\section{TECHNICAL DISCUSSION OF WORK PERFORMED BY ALL PARTIES}

\subsection{BURNER AND GAS FLOW FIELD MODELING AND VALIDATION}

This section documents the investigation of the flow field and combustion modeling for the burners and furnace using pilot testing conducted by Gopher Resource and GTI. This section is broadly divided into two: a description of the mathematical models used within the commercial CFD package STAR-CCM+ [15] (Sec. 3.1.1, Sec. 3.1.2, Sec. 3.1.3) and validation of the combustion data on a test furnace (Sec. 3.1.4). In STAR-CCM+, reacting flows are solved by two approaches: reacting species transport and flamelet. The reacting species transport model, which includes the chemical source term in the conservation equations, was deemed capable of solving for all or major species using well-benchmarked chemical kinetics schemes for OFC.

\subsubsection{Eddy Break-Up Model Description in STAR-CCM+}

The eddy break-up (EBU) model is used for modeling reacting flows (both premixed and non-premixed) with fast chemistry, where the reaction rate is determined by the turbulent mixing timescale [19]. Premixed combustion consists of chemical reactions which occur due to a pre-mixture of the fuel and oxidizer. In the standard EBU model, the reaction rate is limited by the turbulent mixing rate; in the hybrid EBU model which is employed in this work, the reaction rate is limited by the mixing and chemical kinetic timescales. While the EBU model generally works well for simple reaction mechanisms, the complex chemistry method is recommended for introducing detailed chemistry information to the CFD simulation. In the reacting species transport model, conservation equations of mass, momentum, and energy are solved, along with the species transport equation. The discretization method employed in the solver is the finite volume method, which requires the geometry to be meshed (hexahedral, polyhedral, or tetrahedral). The numerical solution is solved at each timestep using an iterative method controlled by a set of relaxation parameters. First- and second-order temporal and spatial numerical discretization can be selected in the STAR-CCM+ input deck to control the accuracy of the numerical solution. In the current study, all the spatial and temporal discretizations use second-order schemes. The conservation equations used in the reaction transport equation model in integral form are presented here, starting with the continuity equation. All the equations shown here are taken from the STAR-CCM+ Theory manual [15].

$$
\frac{\partial \int_{V} \rho d V}{\partial t}+\int_{V} \rho \mathbf{v} \cdot d \mathbf{a}=\int_{V} S_{u} d V
$$

where $t$ is time, $V$ is volume, $\mathbf{a}$ is the area vector, $\rho$ is the density, $\mathbf{v}$ is the velocity vector, and $S_{u}$ is a user-specified source term. The momentum equation in integral form is as follows:

$$
\frac{\partial \int_{V} \rho \mathbf{v} d V}{\partial t}+\oint_{A} \rho \mathbf{v} \otimes \mathbf{v} \cdot d \mathbf{a}=-\oint_{A} p \mathbf{I} \cdot d \mathbf{a}+\oint_{A} \mathbf{T} \cdot d \mathbf{a}+\int_{V} f_{b} d V+\int_{V} s_{u} d V,
$$

where $p$ is pressure, $\mathbf{T}$ is the viscous stress tensor, $f_{b}$ is the body force term, and $s_{u}$ is a user-specified source term. The energy equation in integral form is as follows: 


$$
\frac{\partial \int_{V} \rho E d V}{\partial t}+\oint_{A}\left[\rho H \mathbf{v}_{r}+\mathbf{v}_{g} p\right] \cdot d \mathbf{a}=-\oint_{A} \dot{q}^{\prime \prime} \cdot d \mathbf{a}+\oint_{A} \mathbf{T} \cdot \mathbf{v} d \mathbf{a}+\int_{V} f_{b} \cdot \mathbf{v} d V+\int_{V} S_{E} d V,
$$

where $E$ is the total energy, $H$ is the total enthalpy, $q$ is the heat flux, $\rho$ is the density, $\mathbf{v}$ is the velocity vector, and $S_{E}$ is a source term. Finally, the species transport equation in integral form is as follows:

$$
\frac{\partial \int_{\tilde{V}}\left(\rho Y_{i}\right) d \tilde{V}}{\partial t}+\oint_{A} \rho Y_{i}\left(\mathbf{v}-\mathbf{v}_{g}\right) \cdot d \tilde{\mathbf{a}}=\oint_{A}\left[J_{i}+\frac{\mu_{t}}{\sigma_{t}} \nabla Y_{i}\right] \cdot d \tilde{\mathbf{a}}+\int_{\tilde{V}} S_{Y_{i}} d V,
$$

where $\sim$ represents Favre averaging, $Y_{i}$ is the mass fraction of the $i^{t h}$ species, $J_{i}$ is the diffusion flux, $\mu_{t}$ is the turbulent viscosity, $\sigma_{t}$ is the turbulent diffusivity, and $S_{Y_{i}}$ is the source term from chemical reactions. The source term from chemical reactions is determined as follows:

$$
\tilde{S}=\rho \tilde{r} \frac{Y_{i}^{*}-Y_{i}}{\tau}
$$

where $\tilde{r}$ is the mean reaction rate, $Y_{i}$ denotes the current mass fraction of species $i$ in a mesh cell, and $Y_{i}^{*}$ denotes the mass fraction of species $i$ in a mesh cell at the end of the time integration with timestep $\tau$. For a steady-state solve, the timestep is the residence time in the corresponding mesh cell. The mean reaction rate for the $i^{\text {th }}$ species using the hybrid EBU model is as follows:

$$
r_{i}=-\min \left(\left|r_{i, k i n}\left(\rho, Y_{1}, Y_{2}, \ldots, Y_{N}, T\right)\right|,\left|r_{i, m i x}\right|\right),
$$

where $\min$ is the minimum operator, $r_{i, k i n}$ is the reaction rate due to chemical kinetics, and $r_{i, m i x}$ is the reaction rate due to turbulent mixing. The reaction rate due to turbulent mixing is defined as follows:

$$
r_{i, m i x}=-\frac{\rho}{W_{F}} A_{e b u}\left(\frac{1}{\tau_{t u r b}}\right) \min \left[Y_{F}, \frac{Y_{O}}{s_{O}}\right],
$$

where $W_{F}$ is the molecular weight of fuel, $A_{\text {ebu }}$ is the EBU coefficient, $\tau_{\text {turb }}$ is the turbulent length scale, and $s_{O}$ is the oxidizer to fuel ratio. The turbulent mixing time is defined as follows:

$$
\tau_{\text {turb }}=\frac{\kappa}{\epsilon} \propto \frac{1}{\omega}
$$

where $\kappa$ is the turbulent kinetic energy, $\epsilon$ is the dissipation rate, and $\omega$ is the specific dissipation rate used in the $\kappa-\omega$ turbulence model. Before defining $r_{i, k i n}$ and $s_{O}$, some general nomenclature should be introduced. An elementary chemical reaction is represented as follows:

$$
v_{A}^{\prime} A+v_{B}^{\prime} B+\cdots \frac{k_{f}}{k_{b}} v_{C}^{\prime \prime} C+v_{D}^{\prime \prime} D+\cdots,
$$

where $v$ is the stoichiometric coefficient, ' indicates reactants, " indicates products, $k_{f}$ is the rate constant of the forward reaction, and $k_{b}$ is the rate constant of the backward reaction. With these definitions, $s_{O}$, is as follows: 


$$
s_{O} \equiv \frac{v_{O} m_{O}}{v_{F} W_{F}}
$$

The net stoichiometric coefficient for the $i^{\text {th }}$ species consumed by the reaction, $j$, is given by:

$$
v_{i j}^{\prime \prime}=v_{i}^{\prime \prime}-v_{i}^{\prime}
$$

Similarly, the overall rate constant $k$ of a reaction is defined as follows:

$$
k=k_{f}-k_{b} .
$$

The net reaction rate of species $i, \dot{\omega}_{i}$ (or $r_{i, k i n}$ ), is calculated by taking the sum of the reaction rate $r_{j}$ (for the $j^{\text {th }}$ reaction) over all reactions, where $r_{j}$ is multiplied by the corresponding stoichiometric coefficient. The net reaction rate for the $i^{\text {th }}$ species is given as

$$
\omega_{i}=\frac{\mathrm{d}\left[B_{i}\right]}{\mathrm{d} t}=\sum_{j=1}^{N_{j}} v_{i j} r_{j}
$$

where $r_{j}$ is defined as follows,

$$
r_{j}=k_{f, j} \prod_{i}\left[B_{i}\right]^{v_{j, i}^{\prime}}-k_{b, j} \prod_{i}\left[B_{i}\right]^{v_{j, i}^{\prime \prime}}
$$

and $[B]$ refers to the concentration of a reactant or product in Eq. 9. The order of the reaction, $j$, is given by summing the exponents $v_{j, i}^{\prime}$ or $v_{j, i}^{\prime}$ for the forward and backward reactions, respectively. The order of the reaction must be known a priori because it is determined experimentally. Additionally, a reaction could be first order with respect to, say, species $A$, or second order with respect to species $B$, or a fractional order with respect to species $C$, and so on (using Eq. 9). The reaction scheme used for natural gas (NG) combustion is detailed in the next section. Finally, the rate constant, $k$, is determined using the Arrhenius equation, where data are available for activation energy $E_{a}$, temperature exponent $\beta$, and the pre-exponential factor $A$. The Arrhenius rate constant is given as

$$
k=A T^{\beta} \exp \left(\frac{E_{a}}{R_{u} T}\right)
$$

where $R_{u}$ is the universal gas constant. If reaction rate data are not available, such as for reactions that take place in challenging environments in which measurement is difficult, a user-supplied reaction rate can be given. 


\subsubsection{Chemical Kinetics Scheme for Oxy-Fuel Combustion of Natural Gas}

The chemical kinetics scheme used for oxy-fuel combustion of NG (methane and trace concentrations of ethane and propane) is based on the four-step Jones-Lindstedt (JL) reaction mechanism [20]. A thorough review of the different commonly used global reaction schemes for oxy-NG combustion is provided in the report by Yin [20]. Some of the key points discussed therein are the following. The adiabatic flame temperature in stoichiometry is 3,042 $\mathrm{K}$ for oxy-NG combustion; the actual flame temperature is expected to be lower because of radiative heat losses and non-equilibrium chemical reactions. A high degree of dissociation in oxy-NG flames, which qualifies it as a heat sink, also makes it difficult to measure the free radicals using a gas sampling probe. From a CFD standpoint, the four-step JL global mechanism is deemed to be acceptable in terms of temperature prediction in comparison with a complete reaction scheme because it presents a good balance between accuracy in terms of predicting the key species and computational cost. The reaction mechanisms are shown in the tables that follow for methane (Table 2), ethane (Table 3), and propane (Table 4). Units are in m, s, kmol, J, and K. Note that the exponents of the different species in the reaction rate equation do not necessarily correspond to stoichiometric coefficients, as discussed in the previous section.

Table 2. JL four-step reaction mechanisms for methane with the kinetic rate data

\begin{tabular}{|c|c|c|c|c|c|}
\hline Reaction & Chemical equation & Rate equation $\left[\mathrm{kmol} / \mathrm{m}^{3}-\mathrm{s}\right]$ & A & $\mathrm{b}$ & $\mathrm{E}[\mathrm{J} / \mathrm{mol}]$ \\
\hline 1 & $\mathrm{CH}_{4}+0.5 \mathrm{O}_{2} \longrightarrow \mathrm{CO}+2 \mathrm{H}_{2}$ & $\begin{array}{c}\frac{d\left[\mathrm{CH}_{4}\right]}{d t}= \\
A T^{\beta} \exp \left(\frac{E_{a}}{R_{u} T}\right)^{-} \cdot\left[\mathrm{CH}_{4}\right]^{0.5}\left[\mathrm{O}_{2}\right]^{1.25}\end{array}$ & $4.4 \cdot 10^{11}$ & 0 & $1.26 \cdot 10^{8}$ \\
\hline 2 & $\mathrm{CH}_{4}+\mathrm{H}_{2} \mathrm{O} \longrightarrow \mathrm{CO}+3 \mathrm{H}_{2}$ & $\begin{array}{c}\frac{d\left[\mathrm{CH}_{4}\right]}{d t}= \\
A T^{\beta} \exp \left(\frac{E_{a}}{R_{u} T}\right) \cdot\left[\mathrm{CH}_{4}\right]\left[\mathrm{H}_{2} \mathrm{O}\right]\end{array}$ & $3.0 \cdot 10^{8}$ & 0 & $1.26 \cdot 10^{8}$ \\
\hline 3 & $\mathrm{H}_{2}+0.5 \mathrm{O}_{2} \rightleftharpoons \mathrm{H}_{2} \mathrm{O}$ & $\begin{array}{c}\frac{d\left[\mathrm{H}_{2}\right]}{d t}= \\
A T^{\beta} \exp \left(\frac{E_{a}}{R_{u} T}\right) \cdot\left[\mathrm{H}_{2}\right]\left[\mathrm{O}_{2}\right]^{0.5}\end{array}$ & $2.5 \cdot 10^{16}$ & -1 & $1.67 \cdot 10^{16}$ \\
\hline 4 & $\mathrm{CO}+\mathrm{H}_{2} \mathrm{O} \longleftrightarrow \mathrm{CO}_{2}+\mathrm{H}_{2}$ & $\begin{array}{c}\frac{d[\mathrm{CO}]}{d t}= \\
A T^{\beta} \exp \left(\frac{E_{a}}{R_{u} T}\right) \cdot[\mathrm{CO}]\left[\mathrm{H}_{2} \mathrm{O}\right]\end{array}$ & $2.75 \cdot 10^{9}$ & 0 & $8.4 \cdot 10^{7}$ \\
\hline
\end{tabular}

\subsubsection{Other relevant models used in STAR-CCM+}

Generally, unsteady solvers are preferred for multiphase flow applications because steady-state solvers are often prohibited by the stiff source terms when modeling phase change. The combustion model was first run in steady state to generate a good initial condition for the transient solver. The furnace startup was ignored because the focus of this study is steady-state operations. 
Table 3. JL four-step reaction mechanisms for ethane with the kinetic rate data

\begin{tabular}{|c|c|c|c|c|c|}
\hline Reaction & Chemical equation & Rate equation $\left[\mathrm{kmol} / \mathrm{m}^{3}-\mathrm{s}\right]$ & $\mathrm{A}$ & $\mathrm{b}$ & $\mathrm{E}[\mathrm{J} / \mathrm{mol}]$ \\
\hline 1 & $\mathrm{C}_{2} \mathrm{H}_{6}+\mathrm{O}_{2} \longrightarrow 2 \mathrm{CO}+3 \mathrm{H}_{2}$ & $\begin{array}{c}\frac{d\left[\mathrm{C}_{2} \mathrm{H}_{6}\right]}{d t}= \\
A T^{\beta} \exp \left(\frac{E_{a}}{R_{u} T}\right) \cdot\left[\mathrm{C}_{2} \mathrm{H}_{6}\right]^{0.5}\left[\mathrm{O}_{2}\right]^{1.25}\end{array}$ & $4.4 \cdot 10^{11}$ & 0 & $1.26 \cdot 10^{8}$ \\
\hline 2 & $\begin{array}{c}\mathrm{C}_{2} \mathrm{H}_{6}+2 \mathrm{H}_{2} \mathrm{O} \longrightarrow \\
2 \mathrm{CO}+5 \mathrm{H}_{2}\end{array}$ & $\begin{array}{c}\frac{d\left[\mathrm{C}_{2} \mathrm{H}_{6}\right]}{d t}= \\
A T^{\beta} \exp \left(\frac{E_{a}}{R_{u} T}\right) \cdot\left[\mathrm{C}_{2} \mathrm{H}_{6}\right]\left[\mathrm{H}_{2} \mathrm{O}\right]\end{array}$ & $3.0 \cdot 10^{8}$ & 0 & $1.26 \cdot 10^{8}$ \\
\hline 3 & $\mathrm{H}_{2}+0.5 \mathrm{O}_{2} \rightleftharpoons \mathrm{H}_{2} \mathrm{O}$ & $\begin{array}{c}\frac{d\left[\mathrm{H}_{2}\right]}{d t}= \\
A T^{\beta} \exp \left(\frac{E_{a}}{R_{u} T}\right) \cdot\left[\mathrm{H}_{2}\right]\left[\mathrm{O}_{2}\right]^{0.5}\end{array}$ & $2.5 \cdot 10^{16}$ & -1 & $1.67 \cdot 10^{16}$ \\
\hline 4 & $\mathrm{CO}+\mathrm{H}_{2} \mathrm{O} \longleftrightarrow \mathrm{CO}_{2}+\mathrm{H}_{2}$ & $\begin{array}{c}\frac{d[\mathrm{CO}]}{d t}= \\
A T^{\beta} \exp \left(\frac{E_{a}}{R_{u} T}\right) \cdot[\mathrm{CO}]\left[\mathrm{H}_{2} \mathrm{O}\right]\end{array}$ & $2.75 \cdot 10^{9}$ & 0 & $8.4 \cdot 10^{7}$ \\
\hline
\end{tabular}

Table 4. JL four-step reaction mechanisms for propane with the kinetic rate data

\begin{tabular}{|c|c|c|c|c|c|}
\hline Reaction & Chemical equation & Rate equation $\left[\mathrm{kmol} / \mathrm{m}^{3}-\mathrm{s}\right]$ & A & $\mathrm{b}$ & $\mathrm{E}[\mathrm{J} / \mathrm{mol}]$ \\
\hline 1 & $\begin{array}{c}\mathrm{C}_{3} \mathrm{H}_{8}+1.5 \mathrm{O}_{2} \longrightarrow \\
3 \mathrm{CO}+4 \mathrm{H}_{2}\end{array}$ & $\begin{array}{c}\frac{d\left[\mathrm{C}_{3} \mathrm{H}_{8}\right]}{d t}= \\
A T^{\beta} \exp \left(\frac{E_{a}}{R_{u} T}\right) \cdot\left[\mathrm{C}_{3} \mathrm{H}_{8}\right]^{0.5}\left[\mathrm{O}_{2}\right]^{1.25}\end{array}$ & $4.4 \cdot 10^{11}$ & 0 & $1.26 \cdot 10^{8}$ \\
\hline 2 & $\begin{array}{c}\mathrm{C}_{3} \mathrm{H}_{8}+3 \mathrm{H}_{2} \mathrm{O} \longrightarrow \\
3 \mathrm{CO}+7 \mathrm{H}_{2}\end{array}$ & $\begin{array}{c}\frac{d\left[\mathrm{C}_{3} \mathrm{H}_{8}\right]}{d t}= \\
A T^{\beta} \exp \left(\frac{E_{a}}{R_{u} T}\right) \cdot\left[\mathrm{C}_{3} \mathrm{H}_{8}\right]\left[\mathrm{H}_{2} \mathrm{O}\right]\end{array}$ & $3.0 \cdot 10^{8}$ & 0 & $1.26 \cdot 10^{8}$ \\
\hline 3 & $\mathrm{H}_{2}+0.5 \mathrm{O}_{2} \rightleftharpoons \mathrm{H}_{2} \mathrm{O}$ & $\begin{array}{c}\frac{d\left[\mathrm{H}_{2}\right]}{d t}= \\
A T^{\beta} \exp \left(\frac{E_{a}}{R_{u} T}\right) \cdot\left[\mathrm{H}_{2}\right]\left[\mathrm{O}_{2}\right]^{0.5}\end{array}$ & $2.5 \cdot 10^{16}$ & -1 & $1.67 \cdot 10^{16}$ \\
\hline 4 & $\mathrm{CO}+\mathrm{H}_{2} \mathrm{O} \longleftrightarrow \mathrm{CO}_{2}+\mathrm{H}_{2}$ & $\begin{array}{c}\frac{d[\mathrm{CO}]}{d t}= \\
A T^{\beta} \exp \left(\frac{E_{a}}{R_{u} T}\right) \cdot[\mathrm{CO}]\left[\mathrm{H}_{2} \mathrm{O}\right]\end{array}$ & $2.75 \cdot 10^{9}$ & 0 & $8.4 \cdot 10^{7}$ \\
\hline
\end{tabular}


3.1.3.1 Equation of state The equation of state is a thermodynamic equation that relates density to state variables (pressure and temperature). An equation of state is required for each phase of the multiphase flow model to model the phase density and mixture density variations with respect to pressure and temperature changes. STAR-CCM+ has multiple built-in equations of state and allows for implementation of custom equations of state. Choice of the equation of state is dependent upon flow and operating conditions. In the case of reacting flows for which changes in thermophysical properties and compressibility effects must be considered, the ideal gas was chosen as the equation of state, along with temperature-dependent properties for specific heat at constant pressure, $C_{p}$. For the full-furnace operation, this was slightly tweaked because temperature dependent functions were used for density instead of the ideal gas law. The effect of pressure was deemed to be relatively minor; the pressure change across the furnace did not significantly impact the properties compared to the steep temperature gradients.

3.1.3.2 Turbulent models STAR-CCM+ offers a selection of turbulent models for both single-phase and multiphase flows [15]. For single-phase flow, the turbulent nature of the flow commonly depends on the geometry and the Reynolds number. For multiphase flow, the turbulent nature of the flow is also influenced by the physics themselves, such as reacting flows, phase change, interfacial forces, and so on. The current study concerns a turbulent combustion problem for which the rapid gas expansion in the flame front generates significant turbulent eddies. Menter's shear stress transport k-omega model [21] with an all $\mathrm{y}+$ wall treatment was selected to perform the numerical analysis presented in all sections for the Eulerian gas phase. The shear stress transport k-omega model modifies the standard k-omega model, by adding a blending function that includes a cross diffusion term away from the walls but not near the wall. This retains the advantages of the standard k-omega model in terms of its improved performance for simulating internal flows in comparison to the k-epsilon model, with reduced sensitivity to the free-stream value of $\omega$, as in the standard k-omega model [21].

3.1.3.3 Radiation modeling Volumetric radiation exchange, also called participating media, can be modeled in STAR-CCM+ for radiation heat transfer from various media—such as an Eulerian gas phase, a solid Lagrangian phase, etc. - that can absorb, emit, or scatter thermal radiation. The discrete ordinate method (DOM), which is used in this study, can simulate thermal radiation exchange between diffuse/specular surfaces using the radiative transfer equation. The DOM model requires specification of the number of solid angles into which the sphere surrounding any particular point is discretized. The default "S4 quadrature" is used to balance both accuracy and computational cost. Additionally, the weighted sum of gray gas (WSGG) model is chosen for modeling wavelength-independent spectral properties, and it is widely used in air-fuel combustion processes. WSGG is a reasonable approximation with which to calculate gas mixture radiative properties between a full spectral model and a simple gray gas model, accounting for different gas component absorption coefficients. The total gas absorptivity in the WSGG method (Bouger-Lambert law) is calculated as follows:

$$
\alpha=\sum_{k=0}^{K} a_{k}(1-\exp (-\kappa L))
$$

where $L$ is the optical path length, $\kappa$, is the absorption coefficient of the $k^{\text {th }}$ gas component, $a_{k}$ is the weight factor of the $k^{\text {th }}$ gas component, and $\alpha$ is the total gas absorptivity. The optical path length, which is a user 
input, can range from the cell dimensions to the entire computational domain. The optical path length can be approximated as

$$
L=3.6 \mathrm{~V} / \mathrm{A}
$$

where $V$ is the volume of combustor and $A$ is the surface area.

\subsubsection{Model Validation of Gopher Resource Furnace Measurements}

\subsubsection{Mesh and solver settings}

Once a CFD model has been developed of the geometry and the physics to be solved, the next task consists of verifying and validating the numerical solution using the available experimental data. For validating the combustion simulation, a test furnace was constructed by Gopher Resource as shown in Fig. 4. Tests were conducted for different gas flow rates with Gopher's propriety burners. The furnace construction and measurements are confidential, therefore the details are not provided here. The following experimental measurements were taken for validation:

- Heat loss measurements through the various water-cooled furnace elements

- Temperature measurements at different locations within the furnace

- In-flame CO measurements at various locations within the flame

- Total heat flux and radiative heat flux measurements at different locations

- Flue composition
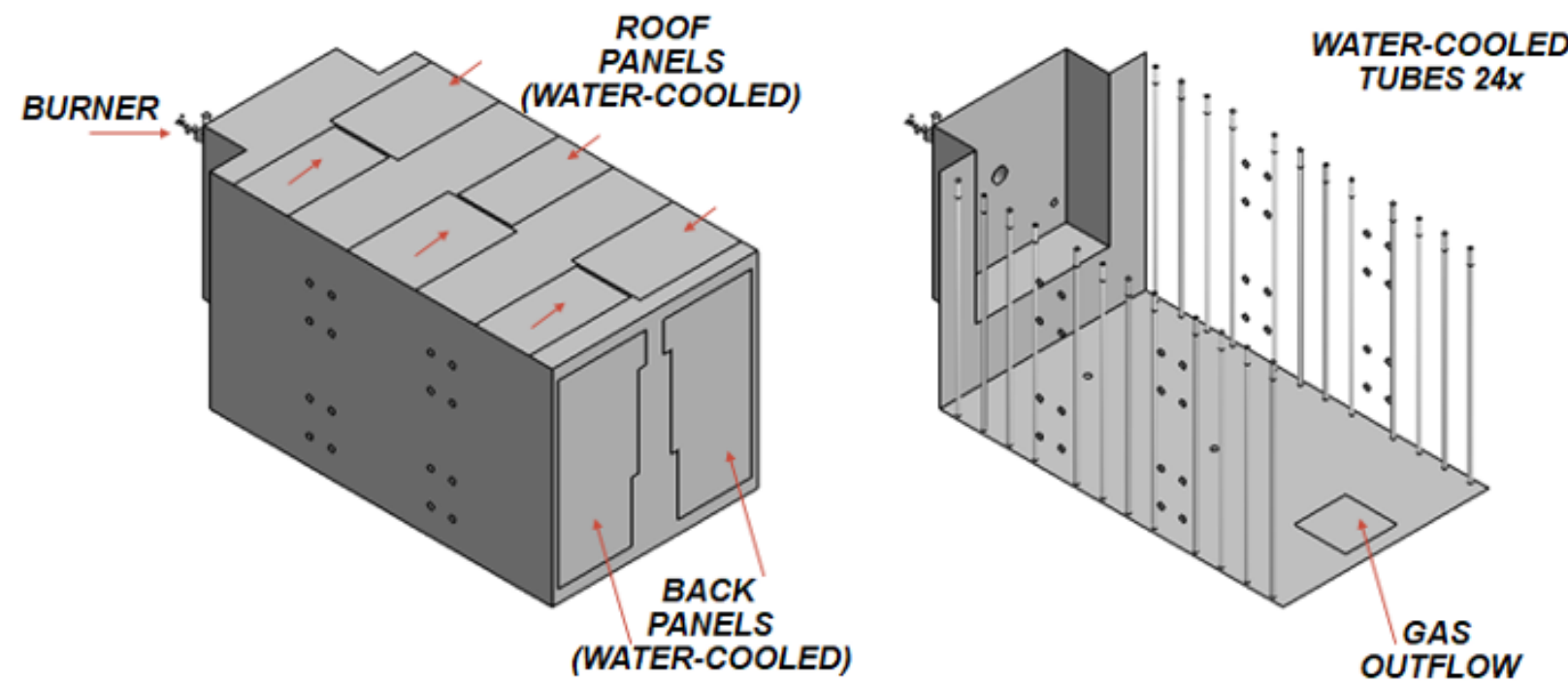

Figure 4. Image of the inside of the test furnace showing views of the cooling tubes, floor plates, and back wall panels.

The choice of the meshing tool is tied to the complexity of the geometry and the assumptions made on the geometry, and this choice can also influence the accuracy of the numerical solution. The CFD model was 
constructed with polyhedral elements given the complexity of the burner, furnace, and cooling tube geometry. STAR-CCM+'s polyhedral mesher has well-documented capabilities for generating complex polyhedral mesh elements with an average of 14 faces from an underlying tetrahedral mesh. More refined and more uniform mesh sizes were used in the burner and the flame locations for better resolution, and a steep change in mesh size away from the peak flame location and toward the back end of the furnace geometry was used. A converged mesh solution consists of mesh generation followed by verification of the numerical solution and then refinement of the mesh until mesh convergence is reached. The process is shown in Fig. 5.

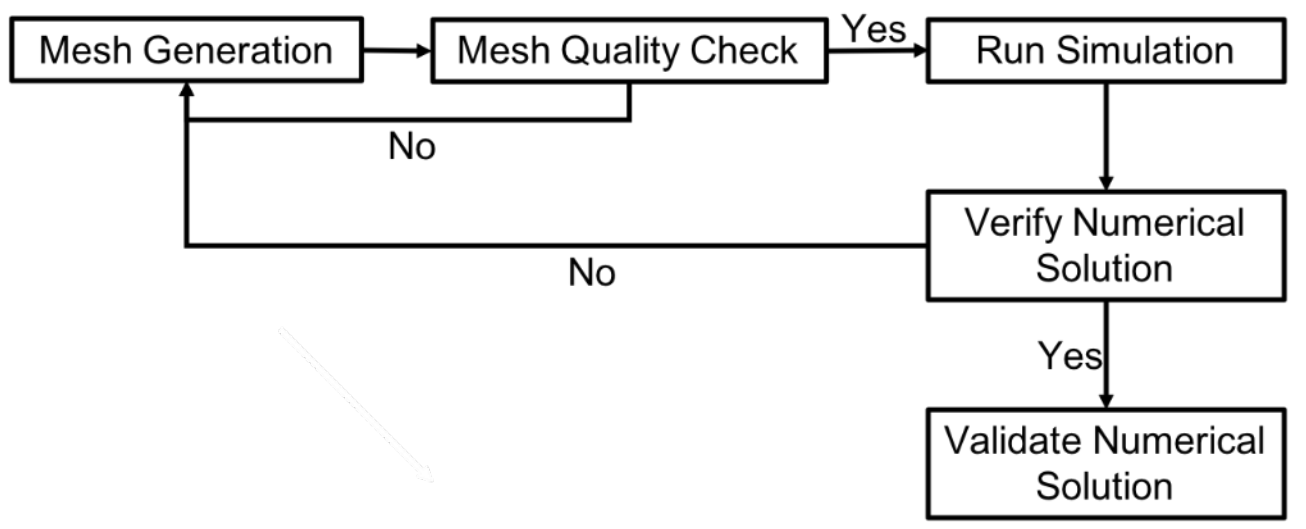

Figure 5. Quality assurance process to optimize the mesh of a CFD model.

The poly and surface remesher mesh settings are described in Table 5. The parameters that are not provided in the table are set to their default values. The boundary layer was resolved with four prism layers of total (relative) widths of $8.125 \%$ of the base size resulting in a wall Y+ of less than 1.0 for the majority of the domain. Note that the size of the cells in the prism layer is bounded by the minimum surface size. A mesh snapshot of the test furnace CFD model is shown in Fig. 6.

Table 5. Mesh settings for the poly and surface remesher models.

\begin{tabular}{lr}
\hline Parameter & Value \\
\hline Meshing tool & Polyhedral and surface remesher \\
Base size & $0.0254 \mathrm{~m}$ \\
Target surface size & $0.0254 \mathrm{~m}$ \\
Minimum surface size & $2.54 \cdot 10^{-5}$ \\
Surface growth rate & 1.5 \\
Total number of cells & $13.5 \cdot 10^{6}$ \\
\hline
\end{tabular}

The EBU model discussed previously was employed to solve for the combustion flow field. The EBU model requires an igniter to activate the combustion model and kick-start the reactions. Note that the reactions themselves progress according to the reaction rates, which are determined by rate-limiting turbulent mixing and chemical kinetic time scales. An EBU igniter was located within the burner, downstream of the inlet tubes, where the fuel and oxidizer were deemed to be well mixed. The location was optimized by initially performing cold flow runs and calculating fuel to air ratios at various locations 


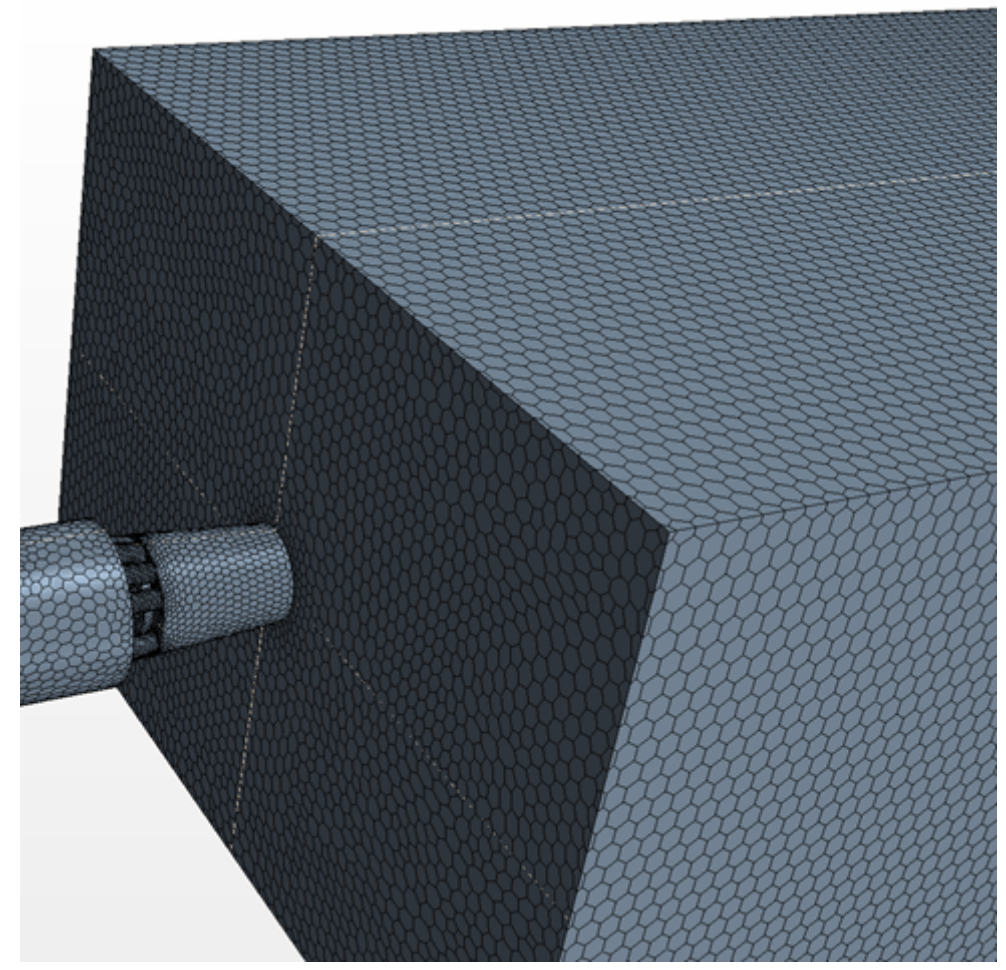

Figure 6. Mesh snapshot of the test furnace showing the well-resolved burner region.

around the region of interest. Reacting flow simulations were also conducted to further optimize the position of the igniter. A snapshot of the spherical igniter region is shown in Fig. 7.

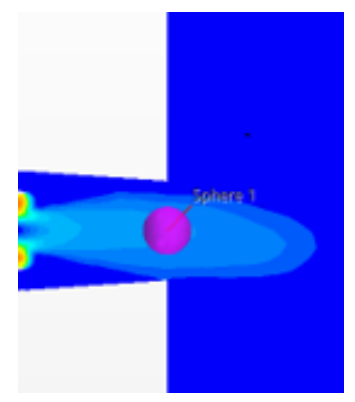

Figure 7. Furnace geometry showing the spherical igniter colored purple.

The coupled solver and turbulence solver models were discussed in the previous section and are omitted here for brevity. STAR-CCM+ automatically enables a set of solvers with parameters to control solver methods and convergence criteria for inner and outer iterations at each timestep. Influence of each solver on the convergence is mostly controlled by relaxation parameters. These relaxation parameters can be tuned to ensure convergence of the solver during the transient runs. The setting of the relaxation parameters highly depends on the physics to be solved, and the values used in our models are summarized in Table 6 .

From a mathematical perspective, initial conditions and boundary conditions are required in a CFD model; 
Table 6. Solver settings for the combustion model.

\begin{tabular}{lr}
\hline Parameter & Value \\
\hline Implicit unsteady & second order \\
Timestep & $1 \cdot 10^{-3} \mathrm{~s}$ \\
Number of inner iterations & 5 \\
Coupled implicit solver: Courant-Friedrichs-Lewy & 0.5 \\
Radiation solver under-relaxation & 0.8 \\
$k-\omega$ Turbulence under-relaxation & 0.5 \\
$k-\omega$ Turbulent viscosity under-relaxation & 0.5 \\
\hline
\end{tabular}

partial differential equations are well posed when they are supplied. Since the experimental data do not contain any transient results, only the steady-state results are of interest for verifying and validating the combustion model. Therefore, initial conditions of the combustion model can be chosen to minimize the central processing unit (CPU) resources (i.e., to recover the steady-state solution in as few timesteps as possible). The initial conditions used in the study are summarized in Table 7. For the species model fraction, the second index in the vector corresponds to nitrogen, and the first index is for the remaining species: fuel components and oxidizer.

Table 7. Initial conditions for the combustion model.

\begin{tabular}{lr}
\hline Parameter & Value \\
\hline Pressure & $1 \mathrm{~atm}$ \\
Velocity & $0 \mathrm{~m} / \mathrm{s}$ \\
Static temperature & $300 \mathrm{~K}$ \\
Turbulence intensity & 0.01 \\
Turbulent velocity scale & $1.0 \mathrm{~m} / \mathrm{s}$ \\
Turbulent viscosity ratio & 10.0 \\
Species mole fraction & {$\left[1 \cdot 10^{-3}, 0.992\right]$} \\
\hline
\end{tabular}

Boundary conditions must be specified to close the mathematical model and mimic the experimental setup. Four types of boundary conditions were used in this combustion model: mass flow rate, pressure outlet, no-slip wall, and interface. The total mass flow rate and the species mole fraction were specified for the gas inlets, and the coolant flow rates were specified for the coolant inlets. For the flue gas and coolant outlets, a pressure outlet condition was used. The no-slip wall condition was selected for the walls. A summary of the boundary conditions is provided in Table 8 . The actual boundary condition values are not provided here because they are proprietary. However, the normalized burner set point for the validation condition is provided in Table 9.

Unlike single-phase CFD models, detecting a steady-state solution when performing a turbulent reacting flow simulation can be quite challenging. Some physical phenomenon are highly unsteady and only achieve a steady-state behavior in a statistical manner. It is then customary to rely on average surface and volume quantities to detect a steady state for reacting flows. Surface-average quantities are commonly used at boundaries to monitor variations as a function of time. For instance, volume-average quantities provide 
Table 8. Boundary conditions for the combustion model.

\begin{tabular}{lr}
\hline Boundary & Boundary type \\
\hline Burner/Gas inlets & mass flow rates of all species \\
Coolant inlets & mass flow rates of coolant \\
Gas outlet boundary & Pressure Outlet \\
Furnace wall boundaries & No-slip wall \\
Inner coolant wall boundaries & No-slip wall \\
Outer coolant wall boundaries & Interface \\
\hline
\end{tabular}

Table 9. Normalized burner set point for the validated combustion model.

\begin{tabular}{lrr}
\hline Flow stream type & Mass flow rate [-] & Temperature [K] \\
\hline Air & 1.0 & $\mathrm{~T}$ \\
Natural gas & 0.176 & $\mathrm{~T}-8$ \\
Primary $\mathrm{O}_{2}$ & 0.261 & $\mathrm{~T}-13$ \\
${\text { Secondary } \mathrm{O}_{2}}$ & 0.113 & $\mathrm{~T}-8$ \\
\hline
\end{tabular}

insight on the bulk flow behavior where the peak flame temperature occurs and provide information on the flame front when tracked as a function of time. Mass conservation between the inlets and outlets is also a good indicator for assessing the convergence of the numerical solution. Temporal variations of the turbulence intensity are commonly used to assess the convergence of the turbulence model. The following metrics were monitored as a function of time for the purpose of assessing the performance of the numerical solution, as well as to to identify when a stationary state was reached:

- Volume-averaged gas mixture temperature

- Volume-averaged mass fraction for key reactant and product species

- Turbulence intensity

- Pressure, temperature, and flue gas mass fraction at outlet boundary

- Conservation of mass flow rates for all species and for the coolant

- Mass imbalance

\subsubsection{Numerical Results and Analysis}

In this section, numerical results for the combustion model of the experimental test furnace are presented and analyzed. All the cases were initialized with the initial conditions listed in Table 7 but with different boundary conditions, which were specified for each case. The numbers of cores utilized for the combustion simulation runs (Libby cluster), and some other parallel processing settings, are shown in Table 10.

To reach a stable steady-state solution, the following workflow was used to minimize CPU resources while conserving the desired degree of accuracy. A coarser mesh was initially used to develop the flow with first-order spatial and temporal numerical methods. Once the numerical solution approached first-order convergence in space, second-order numerical methods were triggered to improve accuracy after the mesh 
Table 10. Parallel processing settings for the lagrangian simulation runs

\begin{tabular}{lr}
\hline Parameter & Value \\
\hline Number of nodes & 2 \\
Number of cores per node & 32 \\
\hline
\end{tabular}

was refined to a desired level. Data were then extracted from specific points in the simulated space to match those from measurements made at Gopher's test facility. The outflow temperature of water from each cooling tube and the centerline temperature of the flue gas along the burner axis were used as metrics for validation. Based on the profiles generated, the model was deemed suitable for use in simulating the OFC of NG in the reverberatory furnace. Flow field validation is shown in Fig. 8. 


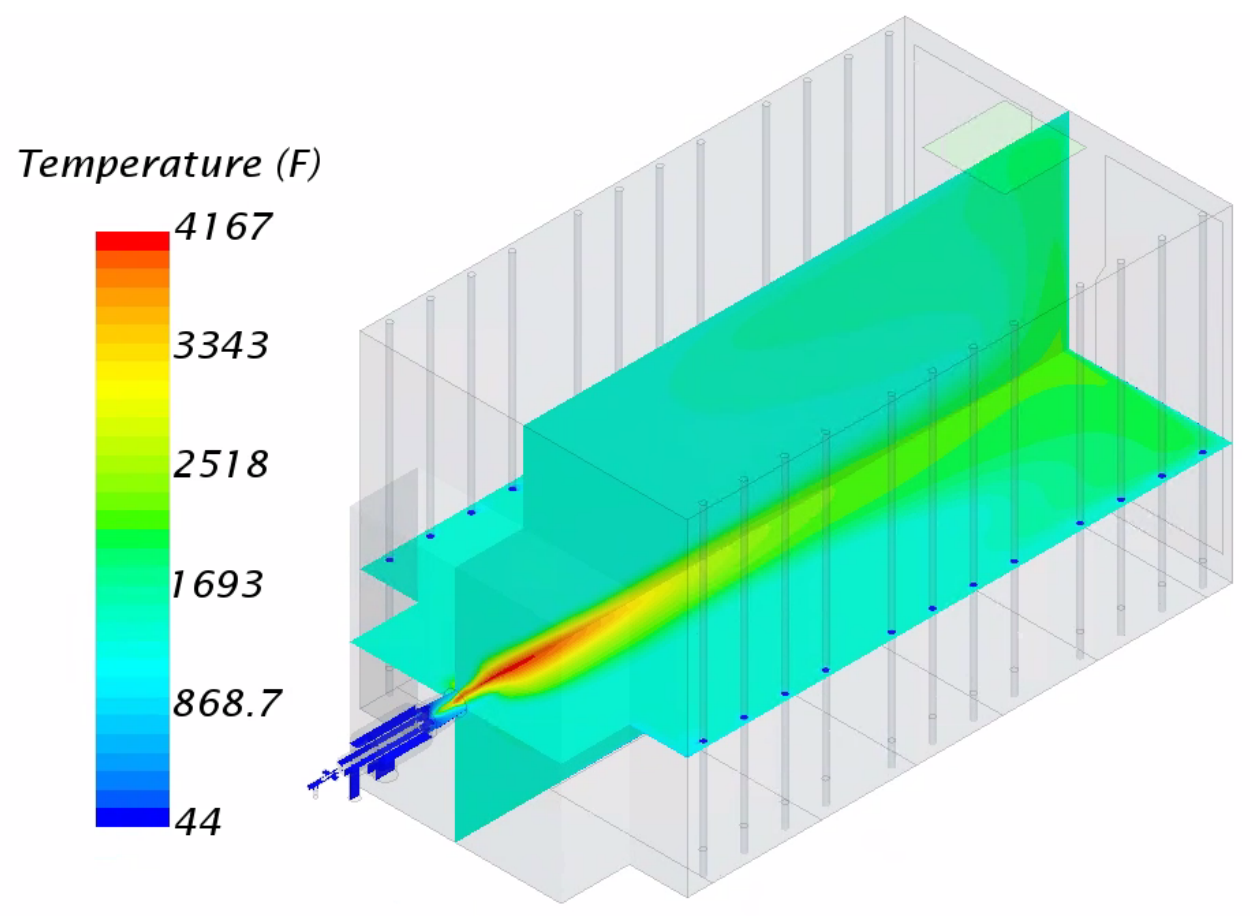

(a) Fluid temperature profiles at two orthogonal cut planes.

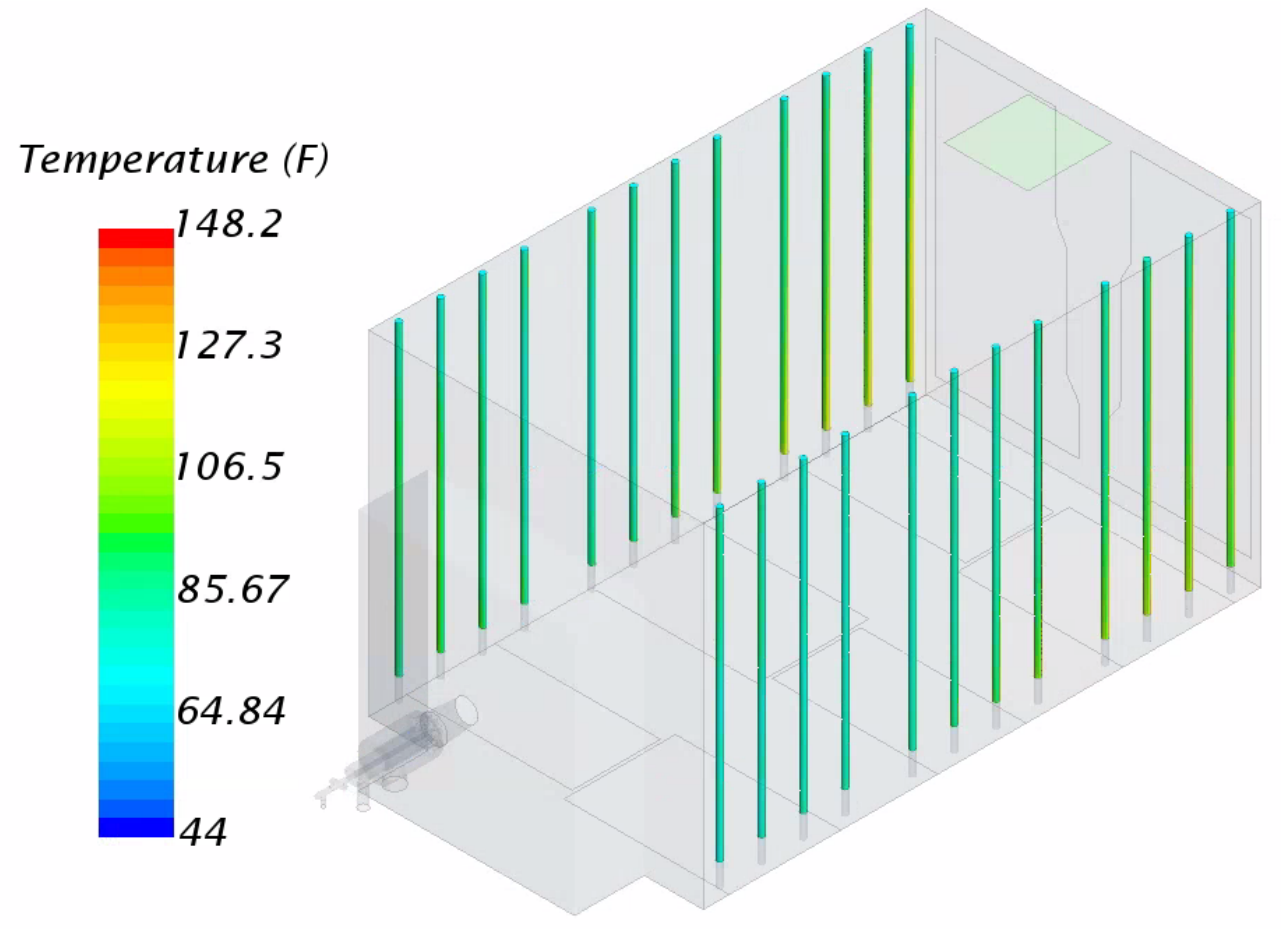

(b) Coolant tube temperature profiles.

Figure 8. Furnace measurement validation and combustion flow field simulation. 
The comparison of simulation results against experimental (normalized) flame temperature and outflow (normalized) coolant temperature is shown in Fig. 9, for a chosen inlet boundary condition. For the measured data set, each "data point" in the plot is calculated as a percentage deviation from the median of the data series and are shown to indicate the variation of the measured values with location. For the simulated comparison (Fig. 9a), the deviation is calculated as simulated to the actual measured value at that location. In summary, the maximum deviation of the simulated flame temperatures is approx. $12 \%$ which is acceptable considering the model approximation and the experimental uncertainties. The simulated coolant outflow temperatures show a higher deviation because there were differences between the actual coolant system and the simulated system, and the actual CAD geometry was not provided.

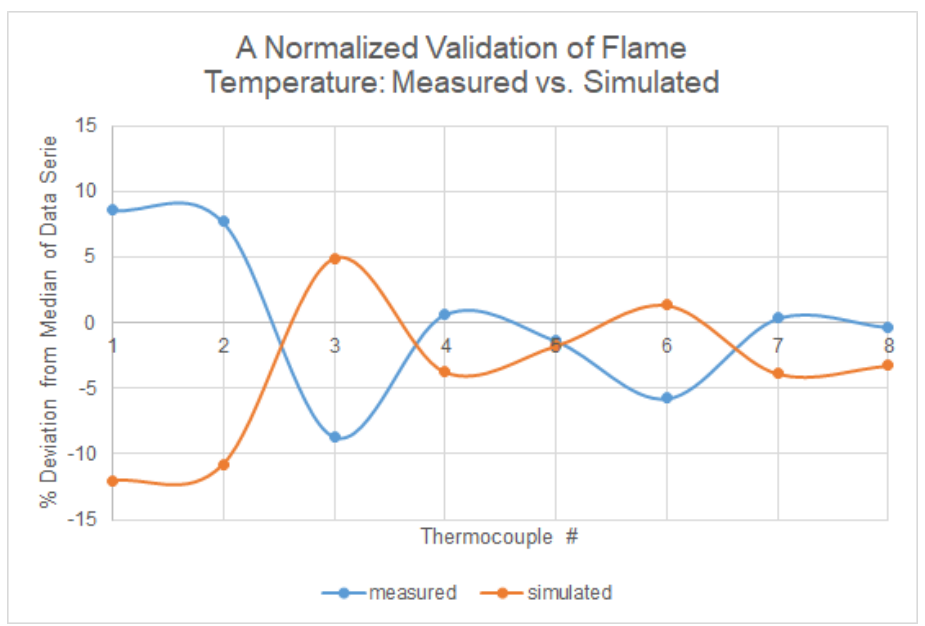

(a) Normalized validation of experimental flame temperatures.

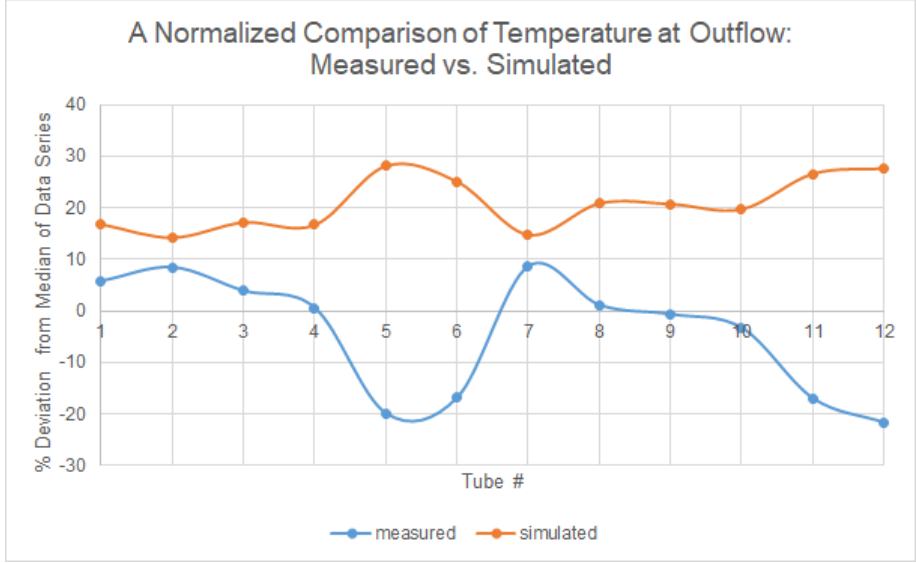

(b) Normalized validation of coolant temperature at outflow.

Figure 9. Validation of experimental data for a chosen condition. 


\subsection{THERMAL AND HYDRODYNAMICS INVESTIGATION OF LAGRANGIAN PARTICLE LOADING}

This section details the mathematical models (Sec. 3.2.1) and physical models (Sec. 3.2.2) used for the Lagrangian multiphase flow (LMP) models within STAR-CCM+ [15]. From a computational perspective, the LMP model is the most suitable to simulate multicomponent feeds. In the Lagrangian framework, the solid feed (material) particles are treated as parcels of mass of a specified shape, using applicable conservation laws including accounting for heterogeneous particle-fluid reactions. Lagrangian models are recommended for particle-laden flows in which the volume fraction of particles is low (less than 10\%) compared with the finite-volume cell. To account for detailed particle-particle interactions, the DEM was used in this study. Although the DEM is restricted to solid particles, it allows for modeling of various shapes. This study is restricted to spherical particles; the effect of particle shape could be investigated in the future. The DEM allows for single- or multicomponent particles. The solid feed for secondary $\mathrm{Pb}$ recovery for the current study has the following constituents: coke $(\mathrm{C})$, lead carbonate $\left(\mathrm{PbCO}_{3}\right)$, lead hydroxide $\left(\mathrm{Pb}(\mathrm{OH})_{2}\right)$, lead oxide $(\mathrm{PbO})$, lead sulfate $\left(\mathrm{PbSO}_{4}\right)$, lead sulfide $(\mathrm{PbS})$, grid $\mathrm{Pb}$, and moisture. Two LMP phases are defined: a single component grid $\mathrm{Pb}$ phase and a multicomponent phase consisting of the remaining $\mathrm{Pb}$ constituents. Moisture is introduced to the solid feed boundary as a component of the Eulerian phase. The coke particles are also introduced as a component in the Eulerian phase; the reasons for this will be highlighted later. Sec. 3.3 details the transfer of Lagrangian feed particles to a continuous fluid caused by smelting/melting physics.

\subsubsection{Lagrangian/DEM Model Description in STAR-CCM+}

The equation of conservation of (linear) momentum for a material or DEM particle of mass $m_{p}$ is given by

$$
m_{p} \frac{\mathrm{d} v_{p}}{\mathrm{~d} t}=\mathbf{F}_{s}+\mathbf{F}_{b}
$$

where $v_{p}$ denotes the instantaneous particle velocity, $\mathbf{F}_{s}$ is the resultant surface forces, $\mathbf{F}_{b}$ is the resultant body forces, and the time derivative is a material/total derivative. In the Lagrangian framework, the state is evolved on a particle by particle basis, whereas the Eulerian fields are frozen. The particle position and particle velocity are obtained by numerically integrating the position/displacement equation with time, as follows:

$$
m_{p} \frac{\mathrm{d} r_{p}}{\mathrm{~d} t}=v_{p}
$$

where $r_{p}(t)$ is the instantaneous position vector at time $t$. In the statistical Lagrangian approach, parcels of particles are tracked. A single integration of the state is applied to all the particles in the parcel, and the interaction term with the Eulerian phase is calculated by multiplying by the number of particles in the parcel. The standard DEM approach tracks individual particles, which includes the results presented in this section. The parcel approach can be used to save computational cost for large particle loadings. In the whole furnace simulation model (Sec. 3.3), the parcel approach is used for the DEM model. For the reminder of this report, particles and parcels will be used interchangeably, unless otherwise specified. The surface forces can be broken down into 


$$
\mathbf{F}_{s}=\mathbf{F}_{d}+\mathbf{F}_{p}+\mathbf{F}_{v m}
$$

where $\mathbf{F}_{d}$ is the drag force, $\mathbf{F}_{p}$ is the pressure gradient force, and $\mathbf{F}_{v m}$ is the virtual mass force. The result of the surface forces is the momentum transferred from the continuous phase to the particle. In the two-way coupling approach used in this study, the result of the $\mathbf{F}_{s}$ is the body force term, which can be similarly decomposed into

$$
\mathbf{F}_{b}=\mathbf{F}_{g}+\mathbf{F}_{c}+\mathbf{F}_{u}+\mathbf{F}_{M R F}+\mathbf{F}_{C o},
$$

where $\mathbf{F}_{g}$ is the gravity force, $\mathbf{F}_{c}$ is the result of all contact forces and is applicable to DEM particles, $\mathbf{F}_{u}$ is the user-defined body force term, $\mathbf{F}_{M R F}$ is applicable for moving reference frames, and $\mathbf{F}_{C o}$ is the Coulomb force. Only the $\mathbf{F}_{g}$ and $\mathbf{F}_{c}$ forces are applicable for this study. DEM particles have angular orientations, and therefore their angular momentum should be conserved as follows:

$$
\mathbf{I}_{p} \frac{\mathrm{d} \omega_{p}}{\mathrm{~d} t}=\mathbf{M}_{b}+\mathbf{M}_{c}
$$

where $\mathbf{I}_{p}$ is the particle moment of inertia described by a second-order tensor, $\omega_{p}$ is the particle angular velocity, $\mathbf{M}_{b}$ is the particle (rotational) drag torque, and $\mathbf{M}_{c}$ is the particle torque caused by contact forces. $\mathbf{M}_{c}$ is defined as follows:

$$
\mathbf{M}_{c}=\sum_{\text {contacts }}\left(\mathbf{r}_{c} \times \mathbf{F}_{c m}+\mathbf{M}_{c m}\right)
$$

where $\mathbf{r}_{c}$ is the position of the vector from the particle center of gravity, and $\mathbf{M}_{c m}$ is the moment that acts on the particle from the rolling resistance contact force that, discussed in Sec. 3.2.2.2.

The particle mass transfer model is used when the dispersed phase is volatile, soluble, or reactive. The particle mass balance equation is given as

$$
\frac{\mathrm{d} m_{p}}{\mathrm{~d} t}=\dot{m}_{p}
$$

where $\dot{m}_{p}$ is the rate of mass transfer to the particle, calculated based on the particle reaction rate in the current study. Mass transfer can occur from the particles to the continuous fluid phase if the two-way coupling is active. The energy conservation equation is given as

$$
m_{p} C_{p} \frac{\mathrm{d} T_{p}}{\mathrm{~d} t}=Q_{t}+Q_{r a d}+Q_{s}+Q_{u}
$$

where $T_{p}$ is the particle temperature, $C_{p}$ is the particle-specific heat at constant pressure, $Q_{t}$ is the rate of particle convective heat transfer, $Q_{\text {rad }}$ is the particle radiative heat transfer, $Q_{s}$ is the source term from particle reactions, and $Q_{u}$ is the user-defined source term and includes the latent heat transfer. $Q_{t}$ is given by the Ranz-Marshall correlation, which is suitable for spherical particles up to an $R e_{p}$ of 5,000. Finally, the radiative heat transfer is given as 


$$
Q_{\text {rad }}=\frac{A_{s}}{4} Q_{a, p}\left(G-4 \sigma T_{p}^{4}\right)
$$

where $A_{s}$ is the particle surface area, $Q_{a, p}$ is the absorption efficiency of the particle, $G$ is the incident radiative heat flux, and $\sigma$ is the Stefan-Boltzmann constant.

As mentioned previously, two-way coupling is used in this study because of heterogeneous particle reactions. With two-way coupling enabled in the LMP model, the effects of the dispersed phase on the continuous phase such as displacement, interphase momentum, mass, and heat transfer are taken into account. The displacement of the continuous phase is accounted for using the total volume fraction $\phi_{c}$ of all Lagrangian phases. The Lagrangian dispersed phase equations, when integrated over a cell, yield the source terms, which are then added to the continuous phase equations. As an example, the source term that is added to the continuous phase continuity equation from all particles in a cell $c$ for an unsteady simulation is as follows

$$
S_{m}=-\frac{1}{\Delta t} \sum_{\pi}\left(\int_{t}^{(t+\Delta t)} \int_{V} \delta\left(\mathbf{r}-\mathbf{r}_{\pi}\right) n_{\phi} \dot{m}_{p} d V d t\right)
$$

where the volume integral is the overall mesh cell, $n_{\pi}$ is the number of particles in parcel $\pi, \delta$ is the Dirac delta function to filter out parcels that are not part of the cell, and $\dot{m}_{p}$ is the mass transfer rate from a single particle. For the source terms that are added to the other fluid conservation equations, please refer to the STAR-CCM+ documentation [15].

\subsubsection{Closure Models and other Relevant Models Used in LMP}

\subsubsection{Fluid-particle interaction closure models}

The drag force is often the principal component of the resultant $\mathbf{F}_{s}$ and is defined as

$$
\mathbf{F}_{d}=\frac{1}{2} C_{d} A_{p}\left|\mathbf{v}_{s}\right| \mathbf{v}_{s}
$$

where $C_{d}$ is the drag coefficient of the particle, $\rho$ is the particle density, $\mathbf{v}_{s}$ is the particle slip velocity, and $A_{p}$ is the projected area of the particle. The particle slip velocity is defined as

$$
\mathbf{v}_{s}=\mathbf{v}-\mathbf{v}_{p},
$$

where $\mathbf{v}$ is the instantaneous velocity of the continuous phase. The drag coefficient, $C_{d}$, is typically

obtained from correlations, and in this study, the Di Felice drag correlation is used [22]. In this formulation, a term accounts for the presence of other particles around a particle. The drag correlation is given as

$$
C_{d}=\left(0.63+\frac{4.80}{\sqrt{\epsilon_{i} R e_{p}}}\right)^{2} \epsilon^{2-\zeta}
$$


where $R e_{p}$ is the particle Reynolds number, and $\epsilon_{i}$ is the void fraction around a particle. The term $\epsilon^{2-\zeta}$ accounts for the enhanced drag caused by the presence of other particles, where $\zeta$ is defined as

$$
\zeta=3.7-0.65 \exp \left[-0.5\left(1.5-\log \left[\epsilon R e_{p}\right]\right)^{2}\right]
$$

Finally, the particle Reynolds number, $R e_{p}$, is defined as

$$
\operatorname{Re}_{p} \equiv \frac{\rho\left|\mathbf{v}_{s}\right| D_{p}}{\mu}
$$

where $D_{p}$ is the particle diameter, $\rho$ is the density of the continuous phase, and $\mu$ is the dynamic viscosity of the continuous phase.

The pressure gradient force, $\mathbf{F}_{p}$, is defined as

$$
\mathbf{F}_{p}=-V_{p} \boldsymbol{\nabla} p_{\text {static }}
$$

where $V_{p}$ is the volume of the particle and $\boldsymbol{\nabla} p_{\text {static }}$ is the gradient of the static pressure in the continuous phase. For DEM particles, the drag torque $\mathbf{M}_{b}$ reduces the difference in the rotational velocities between the continuous fluid and the particle. $\mathbf{M}_{b}$ is defined as

$$
\mathbf{M}_{b}=\frac{\rho}{2}\left(\frac{D_{p}}{2}\right)^{2} C_{R}|\boldsymbol{\Omega}| \boldsymbol{\Omega}
$$

where $C_{R}$ is the rotational drag coefficient [23] and $\mathbf{\Omega}$ is the relative angular velocity of the particle. $\mathbf{\Omega}$ is defined as

$$
\mathbf{\Omega}=\frac{1}{2} \boldsymbol{\nabla} \times \mathbf{v}-\omega_{p}
$$

The particle shear lift force, $\mathbf{F}_{L S}$, acts orthogonally to the relative particle motion and is defined as

$$
\mathbf{F}_{L S}=C_{L S} \frac{\rho \pi}{8} D_{p}^{3}\left(\mathbf{v}_{s} \times \omega\right)
$$

where $\omega$ is the curl of the fluid velocity and $C_{L S}$ is the shear lift coefficient [23].

The gravity force, $\mathbf{F}_{g}$, is defined as

$$
\mathbf{F}_{g}=m_{p} \mathbf{g}
$$

where $\mathbf{g}$ is the gravitational acceleration vector. The final fluid-particle interaction force considered is the turbulent dispersion force. This imparts a fluctuating eddy velocity component to the particle, $\mathbf{v}^{\prime}$, as it passes through a sequence of turbulence eddies while traversing the background turbulent flow field. $\mathbf{v}^{\prime}$ is 
calculated based on fluid turbulence length and time scales. Therefore, the instantaneous particle velocity is defined as

$$
\mathbf{v}_{p}=\overline{\mathbf{v}}+\mathbf{v}^{\prime}
$$

where $\overline{\mathbf{v}}$ is the local Reynolds-averaged (mean) velocity component.

\subsubsection{Interparticle and particle boundary DEM interactions}

In STAR-CCM+, DEM particles are modeled as a soft-particle formulation in which particle-particle overlap is allowed to calculate the contact forces, $\mathbf{F}_{c}$. The particle time scale depends on the max function of three different time scales: Rayleigh wave propagation $\tau_{1}$, the impact duration time $\tau_{2}$, and the particle transit time $\tau_{3} . \tau_{1}$ is the time it would take the Rayleigh wave to propagate across a particle, and it is given as

$$
\tau_{1}=\pi \frac{R}{V_{\text {Rayleigh }}},
$$

where $R$ is half the characteristic minimum dimension of the particle used to calculate its moment of inertia, which is the radius of the sphere for spherical particle, and $V_{\text {Rayleigh }}$ is the Rayleigh wave velocity which depends on material properties and is obtained by solving the wave equation. The maximum timestep cannot exceed a user-input time scale— $t_{\text {scale }}$ times $\tau_{1}$.

A second limiting timestep criterion is calculated using Hertz contact theory and represents the duration of impact of two perfectly elastic bodies, given as

$$
\tau_{2}=2.94\left(\frac{5 \sqrt{2} \pi \phi}{4} \frac{1-v^{2}}{E}\right)^{2 / 5} \frac{R}{\sqrt[5]{v_{\text {impact }}}},
$$

where $E$ is the particle's modulus of elasticity, $\rho$ is the particle density, and $v$ is the Poisson ratio of the particle. Note that the maximum timestep cannot be larger than $0.1 \tau_{2}$.

The final limiting timestep, $\tau_{3}$, is based on the assumption that the particles must not move too far within the timestep. This condition prevents missing contacts between particles and particle-wall interactions. Therefore, each particle is constrained to

$$
\tau_{3}=\frac{R}{v_{\text {particle }}}
$$

where the maximum timestep cannot be larger than $0.1 \tau_{3}$. In most situations, $\tau_{1}$ is the limiting timestep, whereas the other two timesteps typically restrict fast moving particles.

The contact force formulation for DEM in STAR-CCM+ is modeled as a pair of spring-dashpot oscillators-one for the normal force and one for the tangential force. Out of the three available contact models, the Hertz-Mindlin model was chosen for this study. The Hertz-Mindlin model is based on the 
Hertz-Mindlin contact theory, and it is a variant of the nonlinear spring-dashpot contact model. According to this model, the force between two spheres in contact is given by

$$
\mathbf{F}_{\text {contact }}=F_{n} \mathbf{n}+F_{t} \mathbf{t}
$$

where $F_{n}$ and $F_{t}$ are magnitudes of the normal and tangential components, respectively. The normal force, $F_{n}$, is given by,

$$
F_{n}=-K_{n} d_{n}-N_{n} v_{n}
$$

where $K_{n}$ is the spring stiffness in the normal direction, $d_{n}$ is the overlap in the normal direction at the contact point, and $N_{n}$ is the normal damping. $F_{t}$ is calculated similarly, using the tangential spring-dashpot model. The reader should refer to the STAR-CCM+ documentation [15] for details on the definition of the components. This model is also applicable for particle-wall collisions; the second sphere is replaced by the wall surface with an infinite radius. In addition to the contact forces, the rolling resistance model can be selected to supply an additional coefficient of (rolling) resistance, $\mu_{r}$, (the force proportional method) with a default value of 0.001 for $\mu_{r}$. The moment caused by rolling resistance, $\mathbf{M}_{r}$, can be calculated as

$$
\mathbf{M}_{r}=-\left(\mu_{r}\left|F_{n}\right|\left|\mathbf{r}_{c}\right|\right) \frac{\omega_{p}}{\left|\omega_{p}\right|}
$$

where $F_{n}$ is the normal contact force from Eq. (43), $\mathbf{r}_{c}$ is the position vector from the particle centroid to the point of contact, and $\omega_{p}$ is the particle angular velocity parallel to the contact plane.

The final DEM interaction force considered herein is particle conduction through particle-particle and particle-wall contacts. In this study, all walls are taken to be adiabatic, and therefore the particle-wall heat exchange is zero. The particle-particle conduction heat transfer between particles $i$ and $j$ is defined as

$$
q_{i j}=4 r_{c} k\left(T_{j}-T_{i}\right)
$$

where $r_{c}$ is the contact area radius, $k$ is the equivalent (parallel) thermal conductivity, and $T$ is the particle temperature.

\subsubsection{Lagrangian and DEM Modeling Runs}

\subsubsection{Mesh and solver settings}

As mentioned previously, simulations of melting and smelting were decoupled to identify relevant impacts of physics in the solution before integrating models, which will be discussed in Sec. 3.3. The Eulerian combustion model was run to convergence for the actual furnace and then frozen (i.e., the solvers were frozen for combustion and did not run beyond this point). The actual furnace differs from the test furnace in that it has four burners, and the cooling pipes are embedded in the refractory walls and are not considered in the simulation domain. The mesh parameters for the furnace domain are similar to Table 5 . The impact of running the LMP and combustion models simultaneously, as well as its impact on the particle dynamics, is covered in the whole furnace simulation. The DEM model was used to simulate the 
burden as rigid, spherical, coarse-grain particles. The locations at which particles enter the fluid continuum and the manner in which they enter were specified using injectors for each Lagrangian phase. Each injector generated new parcels/particles for a given Lagrangian phase.

The part injector is used to model the particle injection for both the grid $\mathrm{Pb}$ phase and multicomponent $\mathrm{Pb}$ phase (hereinafter called the $\mathrm{Pb}$ mixture phase for simplicity). The part injector generates a cloud of injection points from the geometry of the selected parts. The actual geometry consists of a rotating corkscrew that feeds the secondary $\mathrm{Pb}$ feed to the furnace. The burden inlet was sized to correspond to the actual feed hole. Note that the injector phases need not be physical boundaries because STAR-CCM+ is capable of injecting particles from artificial boundaries as well. For the initial runs, uniformly sized 1 in. particles were injected with corresponding mass flow rates. Since the injection particle velocities are unknown, a sensitivity study was performed and the final chosen velocity that best represents the injection pile is shown here in the results. An additional parcel transfer injector is used to transfer the solid DEM particles to the molten phase. This will be covered as part of the whole furnace simulation.

The under-relaxation parameters and the transient solver settings used for the Lagrangian simulation are summarized in Table 11.

Table 11. Solver settings for the LMP model

\begin{tabular}{lr}
\hline Parameter & Value \\
\hline Implicit unsteady & second order \\
Timestep & $1 \cdot 10^{-3} \mathrm{~s}$ \\
Number of inner iterations & 5 \\
Coupled implicit solver CFL & 0.5 \\
LMP maximum Courant number & 0.35 \\
LMP minimum Courant number & 0.05 \\
LMP two-way coupling under-relaxation & 0.5 \\
LMP maximum void fraction & 0.75 \\
LMP maximum sub-steps & 2000 \\
LMP update frequency timesteps & 5 \\
DEM solver user timescale & 1.0 \\
Radiation solver under-relaxation & 0.8 \\
$k-\omega$ Turbulence under-relaxation & 0.5 \\
$k-\omega$ Turbulent viscosity under-relaxation & 0.5 \\
\hline
\end{tabular}

The initial conditions used in the study for the Lagrangian simulation are summarized in Table 12.

\subsubsection{Numerical results and analysis}

In this section numerical results for the LMP model on the actual furnace are presented and analyzed. All the cases were initialized with the initial conditions Table 12. The numbers of cores utilized for the lagrangian simulation runs (Panacea cluster), and some other parallel processing settings, are shown in Table 13.

Figure 10 shows the view from vertically above the furnace of the initial location of the burden of $\mathrm{Pb}$ particles relative to the impinging flame in 3-D symmetry. Because of the momentum of the flame, the 
Table 12. Initial conditions for the LMP model

\begin{tabular}{lr}
\hline Parameter & Value \\
\hline Pressure & $1 \mathrm{~atm}$ \\
Velocity & $0 \mathrm{~m} / \mathrm{s}$ \\
Static temperature & $300 \mathrm{~K}$ \\
Turbulence intensity & 0.01 \\
Turbulent velocity scale & $1.0 \mathrm{~m} / \mathrm{s}$ \\
Turbulent viscosity ratio & 10.0 \\
Species mole fraction & {$\left[1 \cdot 10^{-3}, 0.992\right]$} \\
\hline
\end{tabular}

Table 13. Parallel processing settings for the lagrangian simulation runs

\begin{tabular}{lr}
\hline Parameter & Value \\
\hline Number of nodes & 8 \\
Number of cores per node & 32 \\
\hline
\end{tabular}

burden begins to displace along the length of the furnace. This was corroborated by physical observations at the Gopher Resource plant in Eagan, MN. 


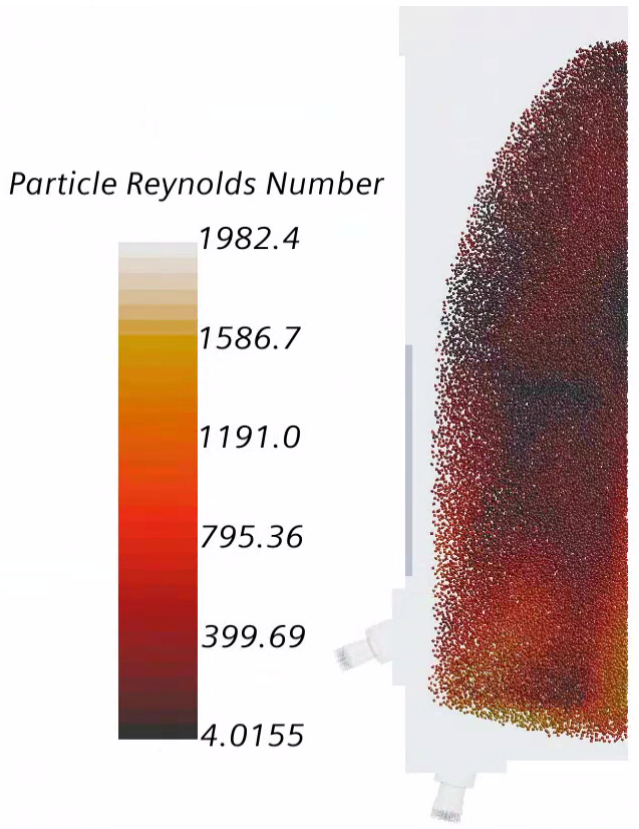

(a) Particle Reynolds Number

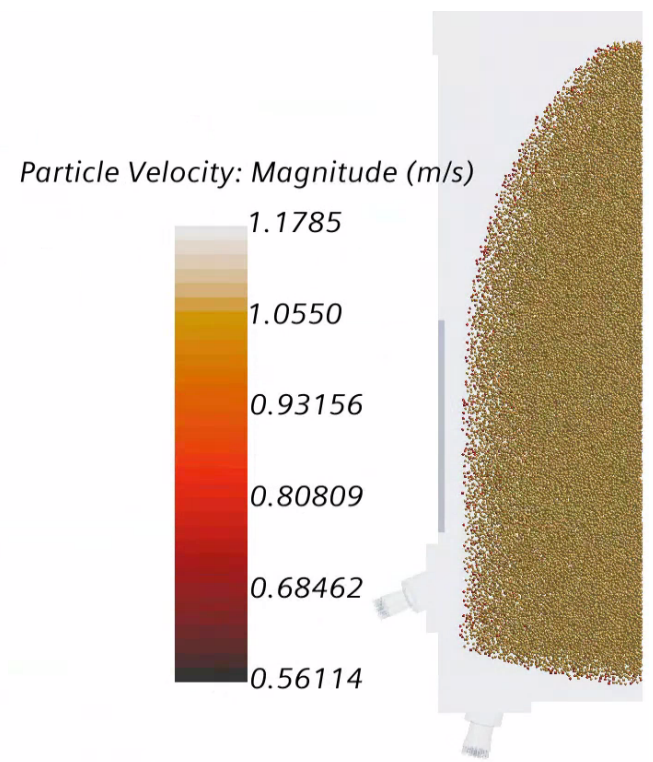

(b) Particle velocity magnitude

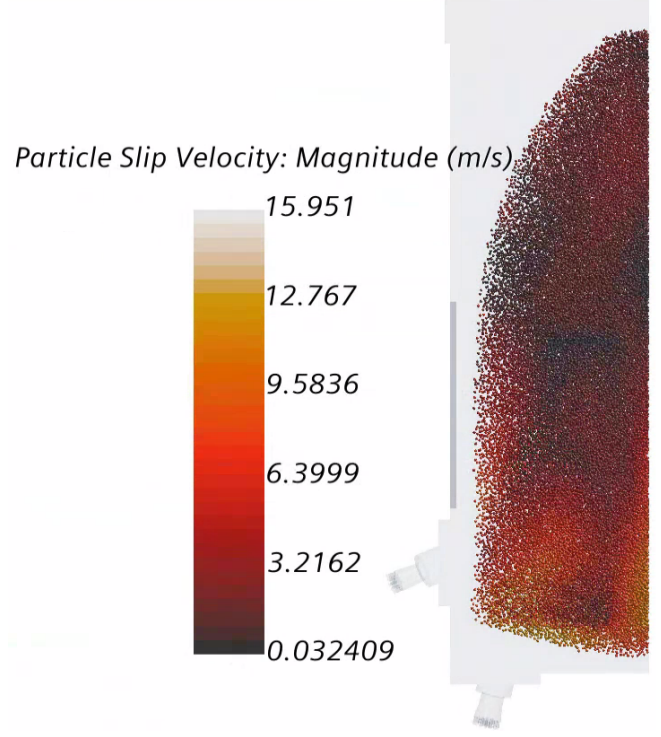

(c) Particle slip velocity magnitude

Figure 10. Cold-flow simulation: (top view) effect of plume momentum on an initially static burden of true composition. 


\subsection{MULTI-PHYSICS SIMULATION OF THE WHOLE FURNACE}

This section details the mathematical models (Sec. 3.3.1) and physical models (Sec. 3.3.2) used within STAR-CCM+ [15] for the whole furnace. From a computational perspective, the whole furnace model is the most challenging to simulate, and it must be noted that there is a lack of studies in the literature looking at integrating all physics into one model. The full-furnace model has three distinct phases: the multicomponent gas phase, the Lagrangian solid feed phase(s), and the molten $\mathrm{Pb}$ phase. The multicomponent gas phase consists of products from the combustion reaction. The solid feed for secondary $\mathrm{Pb}$ recovery for the full simulation comprises of single-component grid $\mathrm{Pb}$ phase and a multicomponent lead mixture phase, similar to the LMP model. Moisture is introduced to the solid feed boundary as a component of the Eulerian phase. Because of the lack of model support for transferring from solid DEM particles to a (continuous) liquid Lagrangian phase for molten $\mathrm{Pb}$, the liquid phase was introduced as a pseudo-gas and was included in the multicomponent gas mixture which participates in gas-phase and gas-solid reactions. The coke particles were also introduced as a component in the multicomponent gas mixture and elaborated in Sec. 3.3.2.

\subsubsection{Relevant Mathematical Models for Full-Furnace Simulation in STAR-CCM+}

\subsubsection{Description of the melting model approximation used in the study}

It was concluded that the current state-of-the-art in validated model sets does not provide for complete resolution of melting phenomena in the presence of heterogeneous chemical reactions. This includes the transition of a material from solid to liquid across the range of solidus and liquidus temperatures, the formation of slurry by solid phase entrained by the molten phase, and the variation in viscosity until the liquidus temperature is reached. The effect of melting solid products is considered via a chemical kinetic scheme in accordance with the Arrhenius model. Although this approach has become a popular one to describing melting kinetics for polymer crystals, as described in the paper by Vyazovkin [24], it would be a first-of-a-kind application for modeling melting in pyrometallurgical furnaces. Unlike in the paper by Vyazovkin [24], the activation energy of the material is treated as zero, and the heat of reaction is treated as equivalent to the latent heat of fusion, $\Delta H_{f}$. The chemical melting model is a reasonable analytical assumption to capture the thermal effects. The validation of the approach is not covered in the report. The validation plan is to test the chemical melting model against the in-built melting model in STAR-CCM+. The test would simulate the melting of a block of ice using VOF, and comparing the energy balance between the two approaches. The melting kinetics for solid $\mathrm{DEM} \mathrm{Pb}$ at its melting point can be described by the following set of equations (refer to Eqs. [13] and [15]):

$$
\begin{array}{r}
\mathrm{Pb}(\mathrm{s}) \stackrel{\Delta H_{f}}{\longrightarrow} \mathrm{Pb}(\mathrm{l}) \\
k=\Delta H_{f}, \\
\omega_{\mathrm{Pb}}=\frac{\mathrm{d}[\mathrm{Pb}]}{\mathrm{d} t}, \\
\therefore \omega_{\mathrm{Pb}} \equiv \Delta H_{f} .
\end{array}
$$

This development was included in the existing model set with the qualified assumption that because there is no chemical change during the melting of a solid, there is next to no activation energy required to break 
chemical bonds. Furthermore, the difference in enthalpy between solid and liquid phases conventionally defines the latent heat of fusion. Thus, thermochemical melting was included as a heterogeneous chemical reaction, and appropriate properties were included for the pseudo-gas(liquid) metal phase.

\subsubsection{Description of the heterogeneous particle reactions used in the study}

The complete set of smelting equations incorporated in the current study is given in Eq. (47). Different smelting reactions take place at different temperatures with varying activation energies and pre-exponent factors for the reaction kinetics. Moreover, there is a paucity in literature on reaction kinetics data for these reactions, and in this study, data from multiple works of literature [25], [26], [27] were used to approximate the rate data, and therefore, optimization studies were performed to deduce the rates from simulations.

$$
\begin{gathered}
\mathrm{PbCO}_{3}(\mathrm{~s}) \longrightarrow \mathrm{PbO}(\mathrm{s})+\mathrm{CO}_{2}(\mathrm{~g}) \\
\mathrm{PbSO}_{4}(\mathrm{~s})+2 \mathrm{C}(\mathrm{s}) \longrightarrow \mathrm{PbS}(\mathrm{s})+2 \mathrm{CO}_{2}(\mathrm{~g}) \\
\mathrm{Pb}(\mathrm{OH})_{2}(\mathrm{~s}) \longrightarrow \mathrm{PbO}(\mathrm{s})+\mathrm{H}_{2} \mathrm{O}(\mathrm{g}) \\
2 \mathrm{PbO}(\mathrm{s})+\mathrm{PbS}(\mathrm{s}) \longrightarrow 3 \mathrm{~Pb}(\mathrm{~s})+\mathrm{SO}_{2}(\mathrm{~g}) \\
2 \mathrm{PbO}(\mathrm{s})+\mathrm{C}(\mathrm{s}) \longrightarrow 2 \mathrm{~Pb}(\mathrm{~s})+\mathrm{CO}_{2}(\mathrm{~g}) .
\end{gathered}
$$

Moreover, there is a limitation on the heterogeneous particle reaction model in STAR-CCM+. The first limitation is that reactions can occur between multiple components within the same Lagrangian and Eulerian phase. This prevents the user from having single-component Lagrangian phases for each $\mathrm{Pb}$ mixture constituent. Secondly, Arrhenius rate kinetics can be used only for single reactant equations. For a reaction with two or more reactants, a user-defined reaction rate should be supplied. This is the reason for introducing the solid coke as an Eulerian gas component with a dummy sublimation reaction of the solid coke to the gaseous phase. This trick allowed the use of Arrhenius rate kinetics for reactions (47b) and (47e). Therefore, the only reaction that required user reaction rates was $(47 \mathrm{~d})$. The mass transfer source term for the $i^{t h}$ species caused by particle reactions is calculated as follows, assuming a first-order rate formulation (Eq. [15]):

$$
\frac{\mathrm{d} m_{i}}{\mathrm{~d} t}=-A T^{\beta} \exp \left(\frac{-E_{a}}{R_{u} T}\right) \alpha_{i} m
$$

where $\alpha_{i}$ is the mass fraction of species $i$. For reaction (47d) with the user reaction rate, the mass consumption rate source term is calculated as follows:

$$
\frac{\mathrm{d} m_{i}}{\mathrm{~d} t}=-r_{u s e r} W_{i} A_{p} v_{i}
$$

where $r_{u s e r}$ is the user reaction rate, $W_{i}$ is the molecular weight of the $i^{\text {th }}$ species, $A_{p}$ is the surface area of the particle, and $v_{i}$ is the stoichiometric coefficient of species $i$. 


\subsubsection{Other Closure Models for the Full-Furnace Simulation in STAR-CCM+}

\subsubsection{Equation of state for the gas-phase components}

The specific heat of the liquid metal was specified using "Polynomial in T" in STAR-CCM+. This allows for the heat of formation of the material to be specified separately, and it thus accounts for the latent heat of fusion. However, the density of the gas mixture is computed as a volume-weighted average, and the ideal gas equation of state computes the density of individual gas components. To incorporate the temperature-dependent density of the liquid metal, the specification of density for the gas mixture was set to "Constant," and the density of the liquid metal was provided as a function of temperature. With this method, the derivative of density was also supplied to ensure an accurate representation of the liquid metal density as a function of temperature.

\subsubsection{Particle clustering for groups of DEM particles}

Based on the initial study, it was estimated that the whole furnace would contain over $1 \cdot 10^{6}$ DEM particles. The clustering particle approach is recommended for industrial flows in which simulations for production facilities can involve particles of various sizes / size distributions, chemical compositions, and temperature-dependent and pressure-dependent properties. This can impose significant computational expenses caused by the associated resolution of interparticle physics for each pair of particle-particle and particle-wall collisions over a given timestep. Although it is essential to resolve all particulate interactions at a level of accuracy commensurate with available computational resources, it may not always be necessary, depending on the nature of the physics. For instance, when particles are initially loaded into the furnace, the slip velocity of particles caused by the flow field and the collisions between particles and with the wall will determine the location of accumulation. Once particles begin to accumulate, the particle bed gains inertia, and the probability of collisions per timestep drastically reduces for older particles.

Therefore, the clustering approach treats agglomerations of particles as a single unit; collisions are no longer critical to determine particle dynamics. This approach conserves the mass of particles and continues to solve the DEM approach with collisions and overlaps between particle pairs [15].

\subsubsection{Whole furnace simulation results}

\subsubsection{Mesh and solver settings}

The Eulerian combustion model was run to convergence for the actual furnace, but it was not frozen, unlike the previously discussed runs. The under-relaxation parameters and the transient solver settings used for the whole furnace simulation are summarized in Table 14.

The initial conditions used in the study for the whole furnace simulation are summarized in Table 15. 
Table 14. Solver settings for the whole furnace model

\begin{tabular}{lr}
\hline Parameter & Value \\
\hline Implicit unsteady & second-order \\
Timestep & $1 \cdot 10^{-2} \mathrm{~s}$ \\
Number of inner iterations & 5 \\
Coupled Implicit solver CFL & 0.5 \\
Radiation solver under-relaxation & 0.8 \\
$k-\omega$ Turbulence under-relaxation & 0.5 \\
$k-\omega$ Turbulent viscosity under-relaxation & 0.5 \\
\hline
\end{tabular}

Table 15. Initial conditions for the combustion model

\begin{tabular}{lr}
\hline Parameter & Value \\
\hline Pressure & $1 \mathrm{~atm}$ \\
Velocity & $0 \mathrm{~m} / \mathrm{s}$ \\
Static temperature & $300 \mathrm{~K}$ \\
Turbulence intensity & 0.01 \\
Turbulent velocity scale & $1.0 \mathrm{~m} / \mathrm{s}$ \\
Turbulent viscosity ratio & 10.0 \\
Species mole fraction & {$\left[1 \cdot 10^{-3}, 0.992\right]$} \\
\hline
\end{tabular}

\subsubsection{Numerical results and analysis}

This section presents and analyzes the numerical results for the full-furnace model on the actual furnace . All the cases were initialized with the initial conditions table 15 . For this simulation, the scalability with number of cores was also investigated because the particle loadings led to $\sim 1 \cdot 10^{6}$ particles with smaller particle sizes than those that were used for the LMP runs. The numbers of cores utilized for the final simulation (Ridge cluster), and some other parallel processing settings, are shown in Table 16.

Table 16. Parallel processing settings for the final whole furnace simulation

\begin{tabular}{lr}
\hline Parameter & Value \\
\hline Number of nodes & 10 \\
Number of cores per node & 128 \\
\hline
\end{tabular}

Profiles from oxy-fuel combustion of NG were provided to Gopher and the normalized combustion gas temperature and velocity profiles are shown in Fig. 11 along burner planes. The location of burners, orientations, and power outputs are specific to the operation of the furnace. 


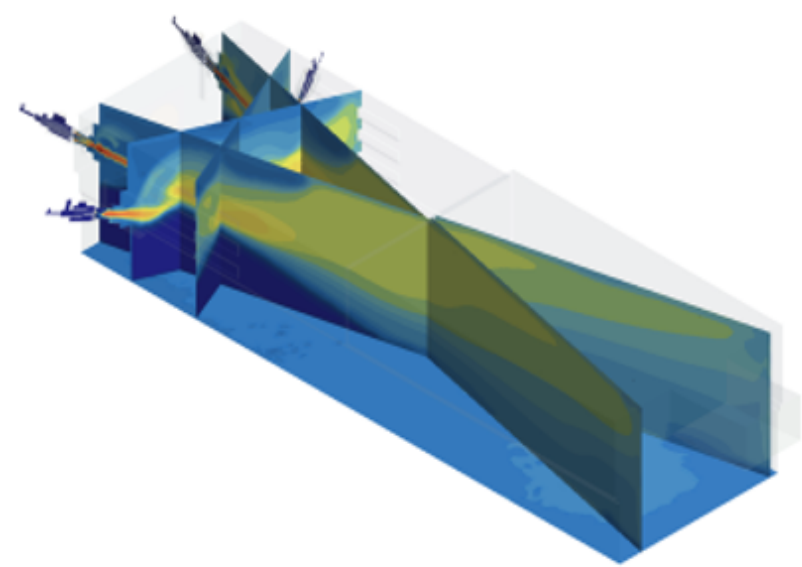

Normalized Temperature $[0,1]$

$\begin{array}{lllllllll}0 & 0.125 & 0.25 & 0.375 & 0.5 & 0.625 & 0.75 & 0.875 & 1\end{array}$

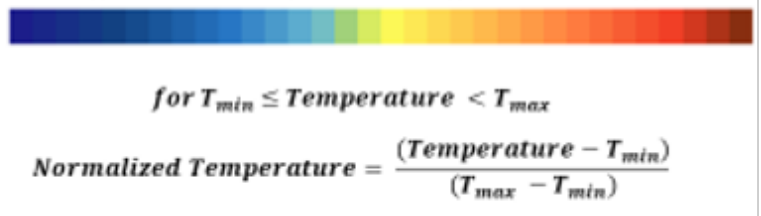

(a) Normalized combustion temperature profiles along different planes.

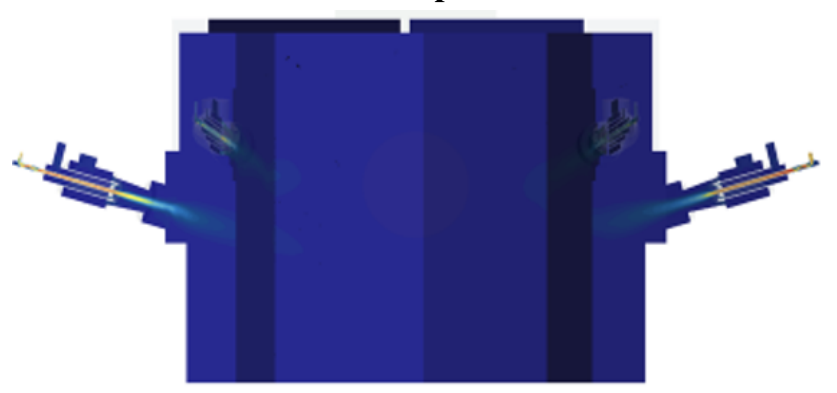

Normalized Velocity $[0,1]$

$\begin{array}{lllllllll}0 & 0.125 & 0.251 & 0.376 & 0.501 & 0.626 & 0.752 & 0.877\end{array}$

Normalized Velocity $=\frac{\text { Velocity }}{\max (\text { Velocity })}$

(b) Normalized combustion gas velocity profiles along different planes.

Figure 11. Normalized combustion model results for the whole furnace steady state operation. 
Particulate profiles are represented to show the impact of the flow field on species behavior. Figs. 12- 15 illustrate the dynamics of particle aggregates (parcels) under the influence of injection into the furnace and thermal effects.

\section{Parcel Index \\ $1.02689 \mathrm{e}+06$ \\ 912789 \\ 798691 \\ 684592 \\ 570494 \\ 456395 \\ 342297 \\ 228198 \\ 114100}

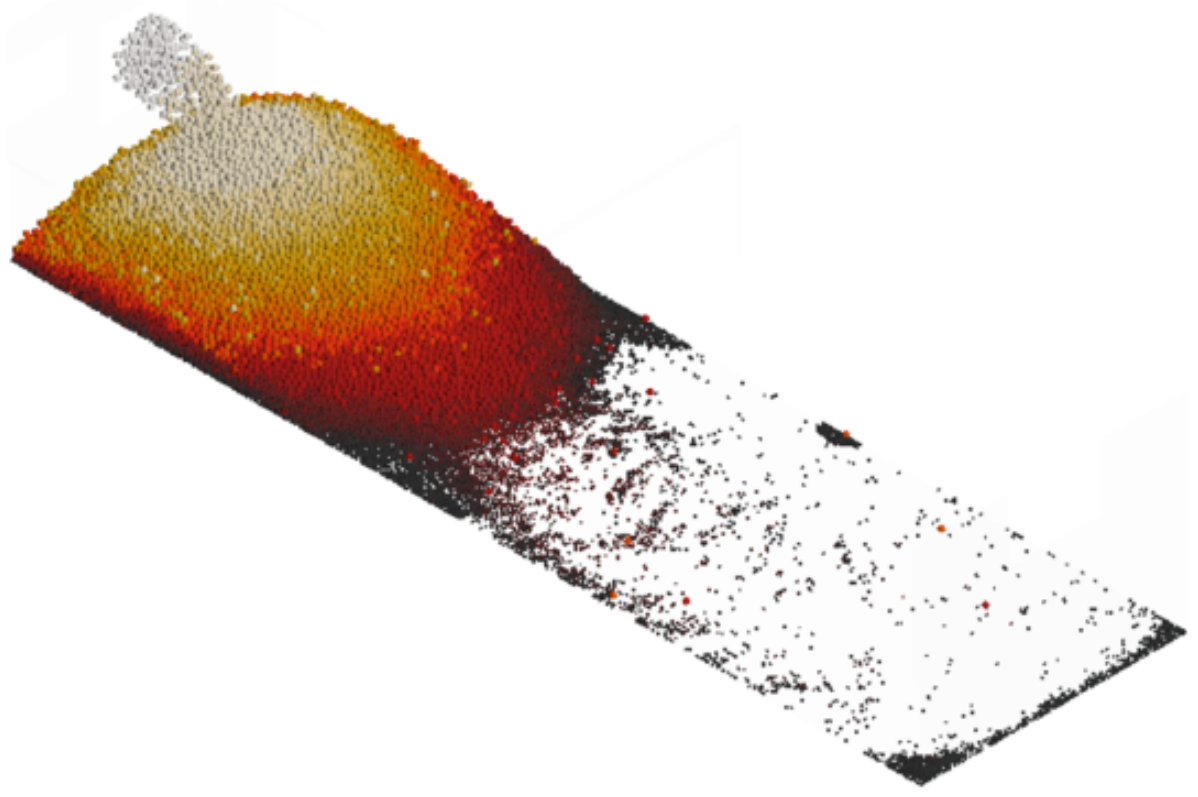

Figure 12. Distribution of parcels in the reverberatory furnace. 

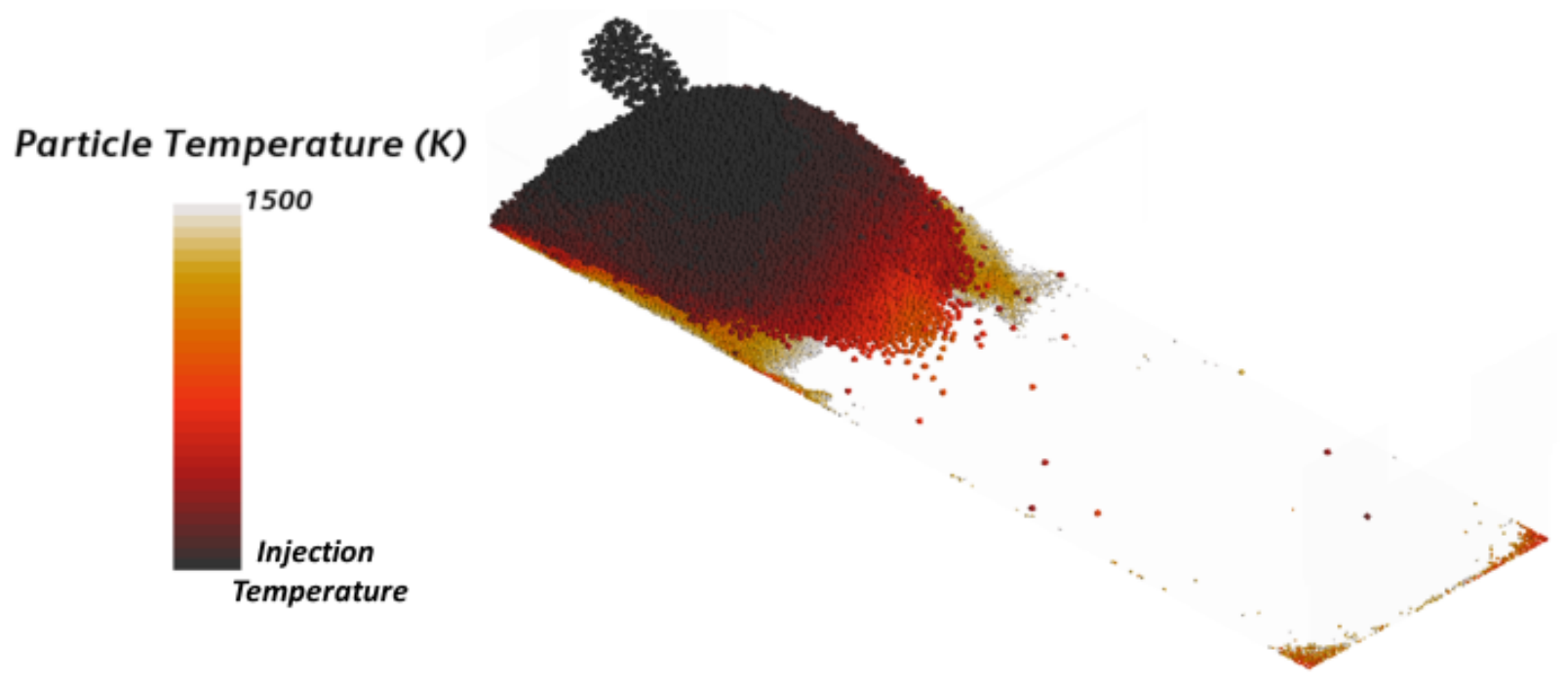

Figure 13. Thermal response of feed to heat from combustion.
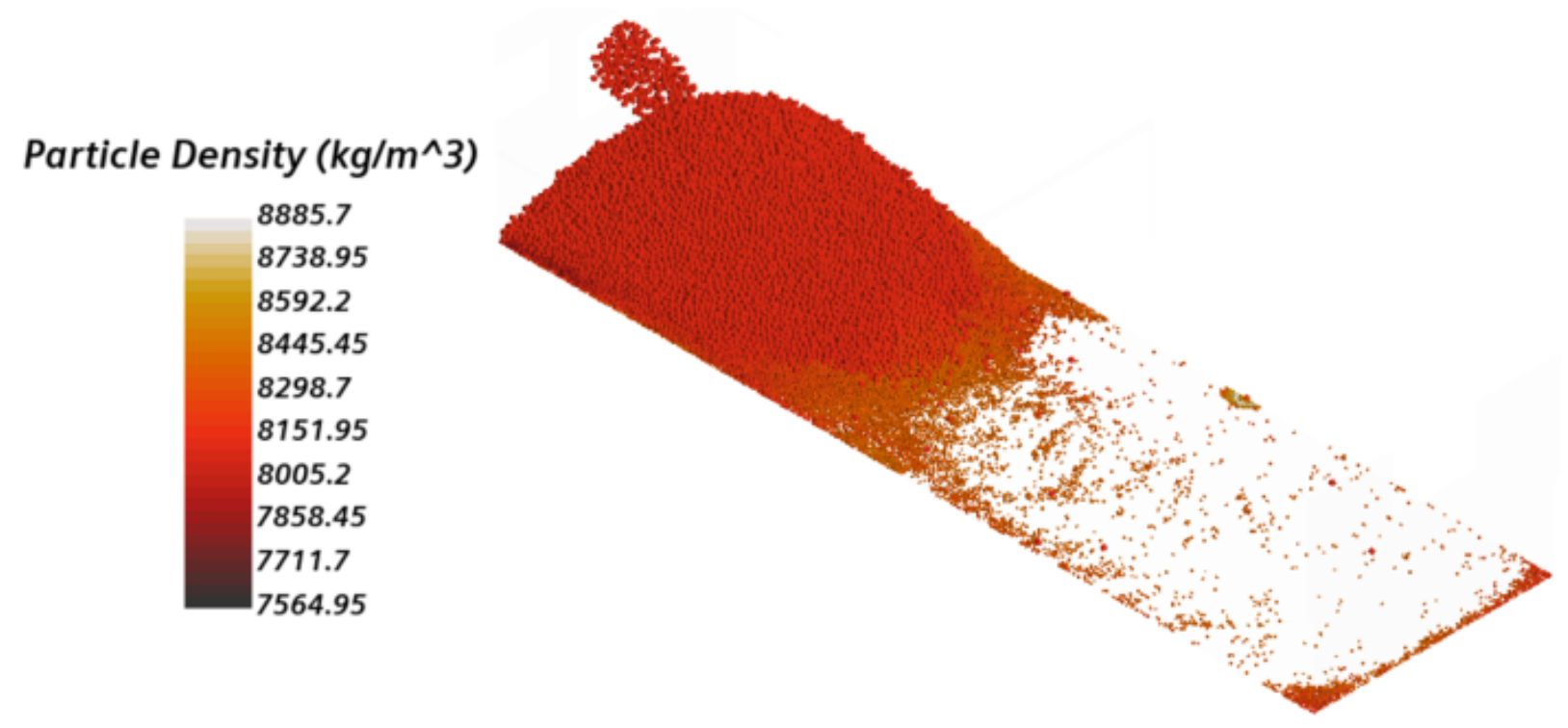

Figure 14. Variation of feed density caused by thermal effects. 

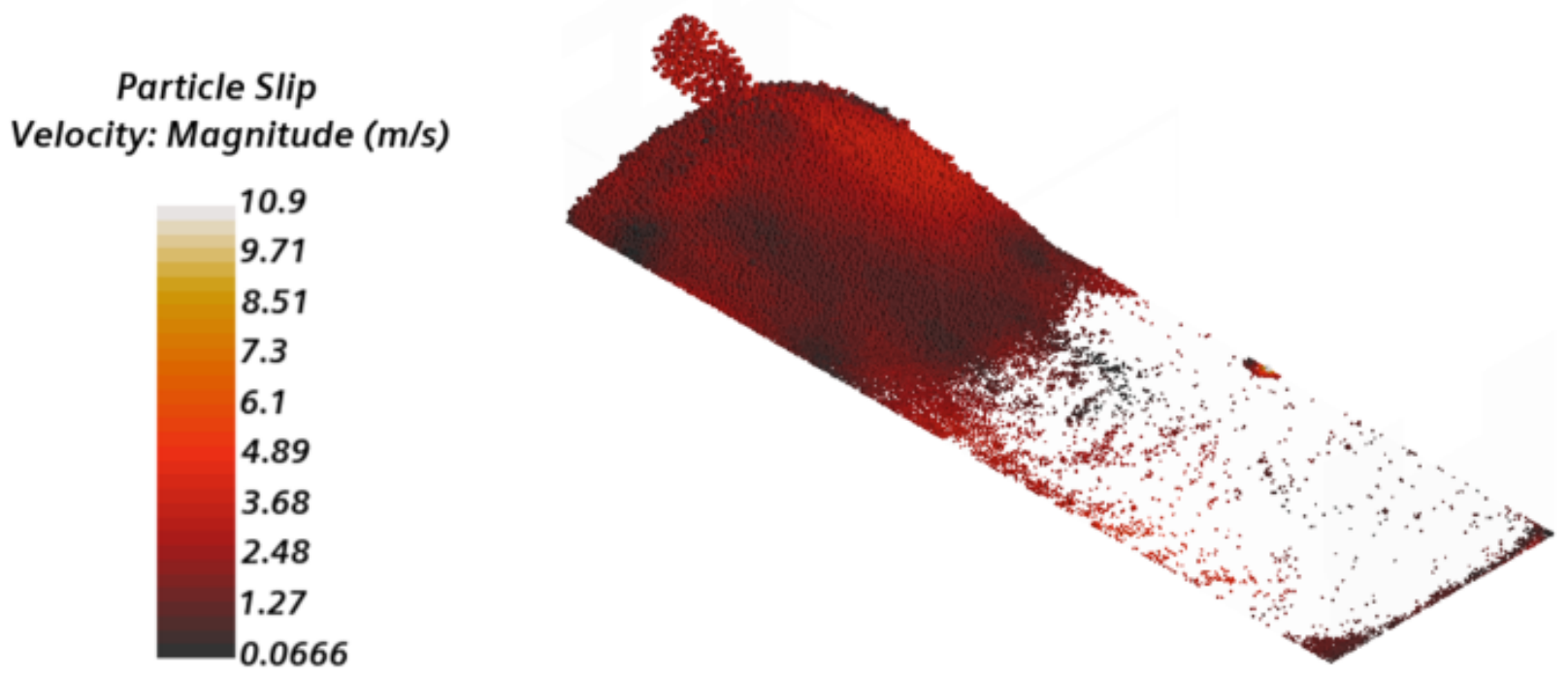

Figure 15. Slip velocity of feed parcels in the flue flow field. 
Fig. 16 shows the variation in species compositions caused by thermal decomposition resulting from heat transfer to the feed from OFC of NG, described in Sec. 3.3.1.2.

The combusting flow field (not shown) imposes high magnitudes of velocity ( $>75 \mathrm{~m} / \mathrm{s}$ ) in the vicinity of the accumulated feed (i.e., burden), which causes a slip with feed parcels which are injected into the flow field. This is evident from Fig. 15, in which parcels injected at $\sim 3 \mathrm{~m} / \mathrm{s}$ reached slip velocities of over $10 \mathrm{~m} / \mathrm{s}$. Such parcels are isolated from the burden because of flocculation and downstream motion from the background momentum of the flue gas. Parcels in the burden gain inertia because of the larger density of the burden (Fig. 14) relative to the flow field and are less affected by ambient turbulence. Thus, the packing of parcels is higher than in the adjacent regions, allowing for convective heat transfer to the exposed parcels and conduction within the burden. Because of this combination of heat transfer modes, the parcels heat up from the bottom of the burden (see Fig. 13), whereas the newer parcels show lower temperatures.

Accordingly, Fig. 16 shows how the species vary in composition as the parcels rise in temperature, absorb heat, and decompose corresponding species. Of the feed components $\mathrm{Pb}, \mathrm{Pb}(\mathrm{OH})_{2}, \mathrm{PbCO}_{3}$, and $\mathrm{PbSO}_{4}$, are the dominant $\mathrm{Pb}$-containing compounds. $\mathrm{Pb}(\mathrm{OH})_{2}$ has the lowest activation energy and was thus the first to decompose fully. $\mathrm{PbCO}_{3}$ requires more energy to decompose and gradually decayed to zero through the burden. $\mathrm{PbSO}_{4}$ is predicted to decompose by chemical reduction with coke in the feed and thus requires good mixing with it to decompose with adequate supply of energy. Lead melts directly upon receipt of latent heat, and this is implemented using the appropriate equation of state for $\mathrm{Pb}(1)$.

Outflow data was relayed to Gopher Resource for comparison with operational data from their plant in Eagan, MN. Relevant metrics include:

- mass outflow of $\mathrm{Pb}, \mathrm{PbO}$, and $\mathrm{PbS}$,

- outflow temperature of flue gas,

- rates of generation of $\mathrm{H}_{2} \mathrm{O}(\mathrm{g})$ and $\mathrm{CO}_{2}(\mathrm{~g})$ from decomposition in the burden, and

- radiant heat flux on the walls of refractory materials.

These comparisons provide a baseline for steady operation of the reverberatory furnace. Future simulations for design improvements, process optimization, and retrofits can be built on the established suite of multiphysics models and meshing strategies employed in the current study. 
Mass Fraction of $\mathrm{Pb}(\mathrm{OH}) 2$
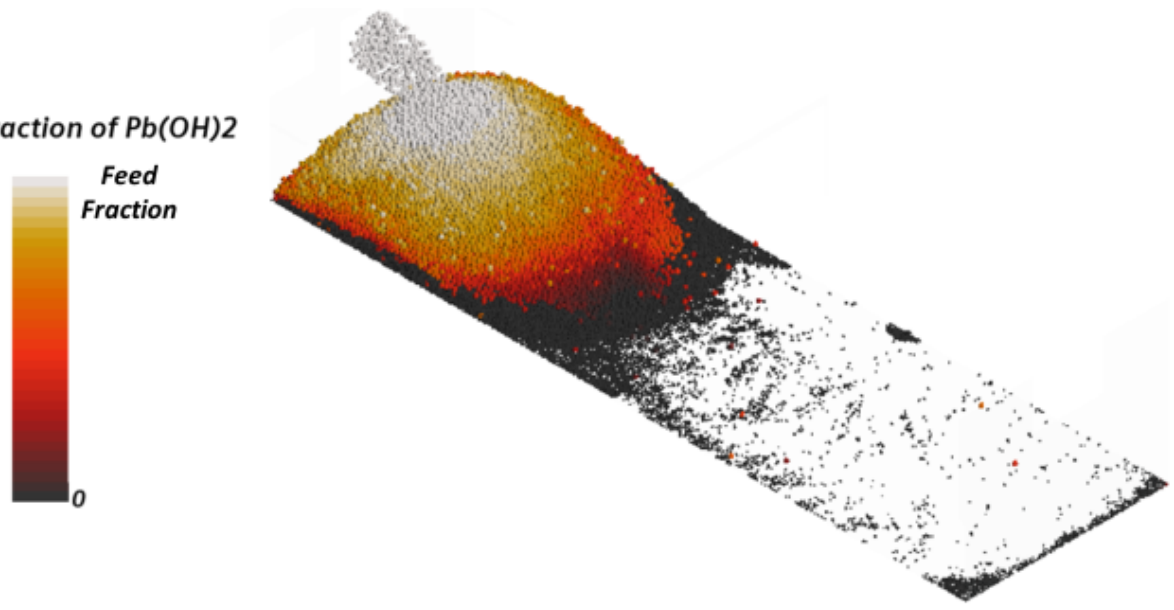

(a) Mass fraction of $\mathrm{Pb}(\mathrm{OH})_{2}$ in the burden.

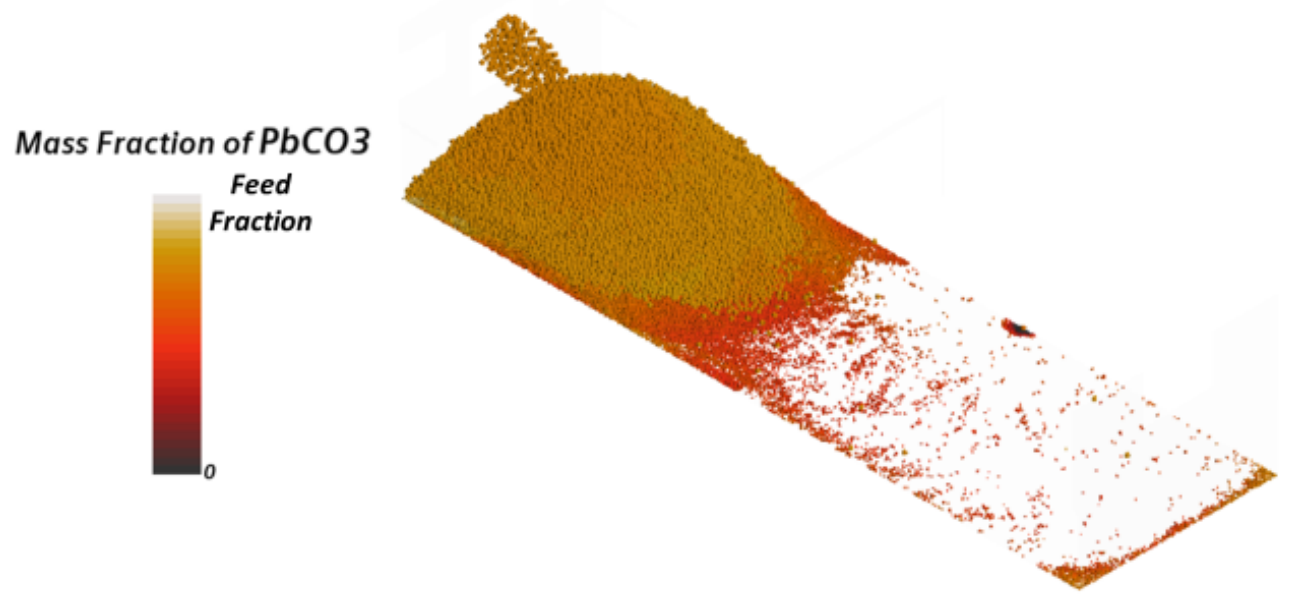

(b) Mass fraction of $\mathrm{PbCO}_{3}$ in the burden.

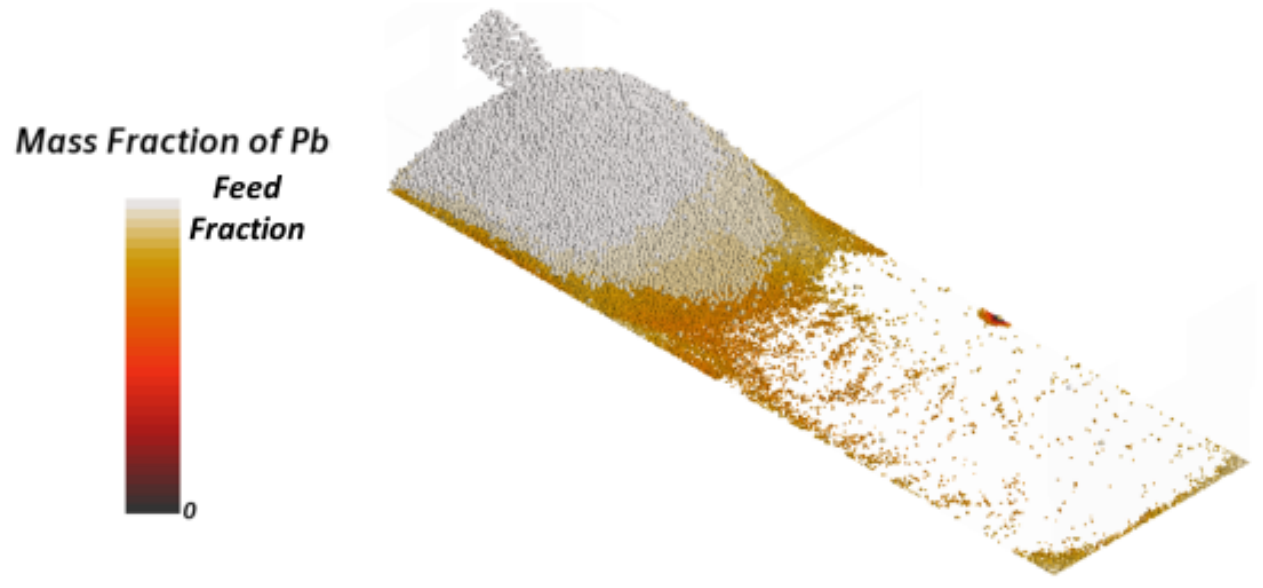

(c) Mass fraction of $\mathrm{Pb}$ in the burden.

Figure 16. Variation of species within parcels due to thermal effects. 


\section{SUBJECT INVENTIONS}

Although the focus of this study is a computational analysis of an existing, operational reverberatory furnace, the scope of invention is restricted to computational efforts. Significant changes were implemented in the numerical scheme within the existing set of commercially available models in STAR-CCM+. The recommended approach from Siemens Product Lifecycle Management (PLM) was used to accomplish many milestones with respect to integration of multiphysics model sets. This study is a first of its kind at this depth of resolution in relevant spatiotemporal scales of pyrometallurgical recovery of nonferrous metals. During this study, the product development team at Siemens PLM were involved with the application at hand, and there is significant potential for future collaboration.

\section{COMMERCIALIZATION POSSIBILITIES}

None.

\section{PLANS FOR FUTURE COLLABORATION}

Future work will likely consist of optimizing the burner design by looking at burner alignment. In general, utilizing the heat from combustion more efficiently and reducing the use of excess oxygen, is typically required in these furnaces. Another area of study would be to look at refractory wear, which is strongly coupled with thermal and viscous stresses from the burner and fluid flow, respectively, and would be optimized to direct maximum heat to the burden and away from the furnace wall. These would lead to improvements in overall furnace energy efficiency. Physics-informed machine learning methods are beginning to be applied to generate surrogates that can be trained on high-resolution codes and used for faster process optimization. Another area of focus would be the need for improvements in the energy resilience of pyrometallurgical furnace systems. In general, upgrading and retrofitting of existing furnaces or new types of furnaces to operate with diverse energy sources-including microgrid connections and coupling to hybrid energy sources for low-load furnace operations-will be required for drastic reductions in $\mathrm{CO}_{2}$ emissions.

As a follow up to this activity, the Oak Ridge National Laboratory (ORNL) team will pursue new strategic partnership opportunities with the US manufacturing industry and will propose new research projects to the DOE Office of Energy Efficiency and Renewable Energy and the DOE Advanced Research Projects Agency. 


\section{SUMMARY}

This report summarizes the work that was performed jointly by ORNL and Gopher Resource and in collaboration with GTI. A three-stage plan was used in completing the project. The plan involved numerous iterations and back and forth between the ORNL and Gopher Resource teams to overcome some of the challenges in tackling such a complex problem. In the first stage, a CFD model of an experimental furnace was constructed in STAR-CCM+ to test Gopher's proprietary burners on an experimental furnace. The measurements included temperature measurements at various locations in the furnace, in-flame $\mathrm{CO}$ measurements, cooling water measurements, etc., and the validated model predicted the measurements with a reasonably good accuracy. In the second stage of the project, the thermal and hydrodynamics of the solid feed particles were investigated using the DEM model. Various particle injection velocities were investigated to replicate the pile loading in the actual furnace. Scalability was also tested with the load balancing option enabled for the Lagrangian solver. Given that the whole furnace simulation was to be run with $\sim 1 \cdot 10^{6}$ particles, these tests were critical to optimize the transient solver settings for the final integrated runs. The third and final stage of the modeling effort focused on simulating the whole-furnace operation which included the combustion flow field, solid particle loading, smelting and melting processes, and formation of slag and $\mathrm{Pb}$. Multiple challenges were overcome, and principal among them was the lack of chemical kinetics data for the smelting reactions and lack of compatibility between Lagrangian particles and STAR-CCM+ melting model. These were overcome with unique solutions such as using employing the thermochemical-based melting model. The formation of molten $\mathrm{Pb}$ that is produced from the smelting reactions, was observed to be consistent with the actual furnace, considering the approximations used in the model. Moving forward, finite volume-based commercial CFD solvers in general will require improvement in the areas of: enhanced multiphase model compatibility, especially for transferring components from Lagrangian to Eulerian phases; support for heterogeneous reaction kinetics across different (appropriate) multiphase models; and Lagrangian particle-based melting models.

This project required intensive use of HPC resources to develop, verify, and validate the CFD combustion model on a test furnace and then sequentially develop a model for the whole-furnace simulation. It is estimated that 40,000 CPU hours were consumed to complete this project on ORNL midsize clusters (Apollo, Libby, Ridge, and Panacea). The scalability and high-quality user interface of the commercial STAR-CCM+ CFD package were essential for the success of this project. Over the course of the project, the ORNL team developed valuable skills for the simulation of unique, multicomponent, multiphase flow systems and gained experience with simulating hard to model pyrometallurgical furnace systems by efficiently utilizing the available computational resources. 



\section{BIBLIOGRAPHY}

\section{References}

[1] T. WATARI, K. NANSAI, and K. NAKAJIMA, "Major metals demand, supply, and environmental impacts to 2100: A critical review," Resources, Conservation and Recycling, 164, 105107 (2021).

[2] U. G. SURVEY, “Mineral Commodity Summaries,” Tech. rep., U.S. Geological Survey (2021).

[3] A. ELSHKAKI, T. GRAEDEL, L. CIACCI, and B. K. RECK, "Resource demand scenarios for the major metals," Environmental science $\mathcal{E}$ technology, 52, 5, 2491-2497 (2018).

[4] I. LEAD and Z. S. GROUP, “The World Lead Factbook," Tech. rep., International Lead and Zinc Study Group (2019).

[5] J.-D. LI, Z. PING, L. ZHOU, L.-Y. CHAI, L. ZHANG, ET AL., "CFD modelling and optimization of oxygen supply mode in KIVCET smelting process," Transactions of Nonferrous Metals Society of China, 29, 7, 1560-1568 (2019).

[6] P. E. KING, M. HAYES, T. LI, Q. HAN, M. HASSAN, and B. GOLCHERT, "Design and operation of an experimental reverberatory aluminum furnace,” Tech. rep., Albany Research Center (ARC), Albany, OR (2005).

[7] C. MEI, X. PENG, P. ZHOU, J. ZHOU, and N. ZHOU, Simulation and optimization of furnaces and kilns for nonferrous metallurgical engineering, Springer (2010).

[8] E. JAK, "Modelling metallurgical furnaces-making the most of modern research and development techniques," in "Extraction 2018," Springer, pp. 103-125 (2018).

[9] M. LAPUTKA and W. XIE, "A Review of Recent Advances in Pyrometallurgical Process Measurement and Modeling, and Their Applications to Process Improvement," Mining, Metallurgy $\mathcal{F}$ Exploration, pp. 1-31 (2021).

[10] D. C. R. ESPINOSA, A. M. BERNARDES, and J. A. S. TENÓRIO, "An overview on the current processes for the recycling of batteries," Journal of Power Sources, 135, 1-2, 311-319 (2004).

[11] J. J. MOORE, Chemical metallurgy, Elsevier (2013).

[12] P. B. QUENEAU, R. LEIBY, and R. ROBINSON, "Recycling lead and zinc in the United States," World of Metallurgy-ERZMETALL, 68, 149 (2015).

[13] A. ANDERSON, J. GROGAN, G. BOGIN, and P. TAYLOR, "Computational Modeling of a Secondary Lead Reverberatory Furnace: Effect of Burden Geometry," in "Extraction 2018," Springer, pp. 881-890 (2018).

[14] A. ANDERSON, Computational fluid dynamic modeling of a secondary lead reverberatory furnace, Ph.D. thesis, Colorado School of Mines (2017).

[15] SIEMENS, "STAR-CCM+ Simcenter Documentation 2021.1," (2021).

[16] V. M. RAO, M.-O. G. DELCHINI, P. K. JAIN, and M. T. BANI AHMAD, "High-Performance Computing to Enable Next-Generation Low-Temperature Waste Heat Recovery," in "ASME Power Conference,” American Society of Mechanical Engineers (2020), vol. 83747, p. V001T11A002. 
[17] L. C. CAPOZZI, A. A. BARRESI, and R. PISANO, "Supporting data and methods for the multi-scale modelling of freeze-drying of microparticles in packed-beds," Data in brief, 22, 722-755 (2019).

[18] L. C. CAPOZZI, A. A. BARRESI, and R. PISANO, "A multi-scale computational framework for modeling the freeze-drying of microparticles in packed-beds," Powder Technology, 343, 834-846 (2019).

[19] B. F. MAGNUSSEN and B. H. HJERTAGER, "On mathematical modeling of turbulent combustion with special emphasis on soot formation and combustion," in "Symposium (international) on Combustion," Elsevier (1977), vol. 16, pp. 719-729.

[20] C. YIN, "Advanced modeling of oxy-fuel combustion of natural gas," Tech. rep., Aalborg Univ. Dept. of Energy Technology, Aalborg (Denmark) (2011).

[21] F. R. MENTER, “Two-equation eddy-viscosity turbulence models for engineering applications," AIAA journal, 32, 8, 1598-1605 (1994).

[22] R. DI FELICE, "The voidage function for fluid-particle interaction systems," International journal of multiphase flow, 20, 1, 153-159 (1994).

[23] M. SOMMERFELD, "Theoretical and experimental modelling of particulate flows," Lecture series, 6, 3-7 (2000).

[24] S. VYAZOVKIN, "Power law and Arrhenius approaches to the melting kinetics of superheated crystals: Are they compatible?" Crystal Growth E Design, 18, 11, 6389-6392 (2018).

[25] S. J. MERRICK, A study of the" roast-reaction" of lead sulfide, Ph.D. thesis, University of British Columbia (1957).

[26] P. J. SLEEMAN, Oxidation of lead sulphide, Ph.D. thesis, University of Plymouth (1976).

[27] S. MAŁECKI, "Thermogravimetric study of the reduction of basic lead sulphate," Journal of Thermal Analysis and Calorimetry, 117, 3, 1091-1095 (2014). 